\title{
Critical phenomena in gravitational collapse
}

\author{
Carsten Gundlach \\ School of Mathematics, University of Southampton, \\ Southampton SO17 1BJ, UK \\ email: cjg@soton.ac.uk \\ http://www.soton.ac.uk/ cjg \\ José M. Martín-García \\ Instituto de Estructura de la Materia, Centro de Física Miguel A. Catalán, \\ C.S.I.C., Serrano 123, 28006 Madrid, Spain \\ email: jmm@iem.cfmac.csic.es \\ http://metric.iem.csic.es/Martin-Garcia/
}

\begin{abstract}
As first discovered by Choptuik, the black hole threshold in the space of initial data for general relativity shows both surprising structure and surprising simplicity. Universality, powerlaw scaling of the black hole mass, and scale echoing have given rise to the term "critical phenomena". They are explained by the existence of exact solutions which are attractors within the black hole threshold, that is, attractors of codimension one in phase space, and which are typically self-similar. Critical phenomena give a natural route from smooth initial data to arbitrarily large curvatures visible from infinity, and are therefore likely to be relevant for cosmic censorship, quantum gravity, astrophysics, and our general understanding of the dynamics of general relativity.
\end{abstract}

\section{Contents}

1 Introduction 2

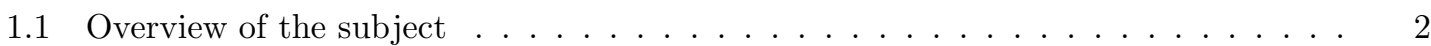

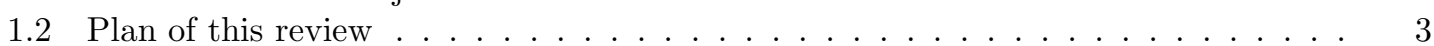

2 Theory 4

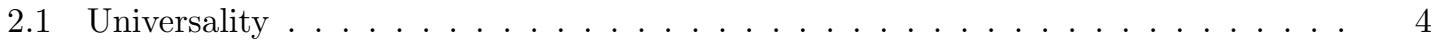

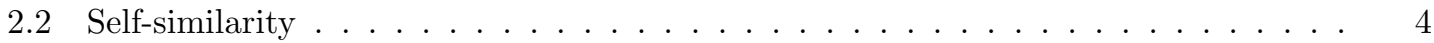

2.3 Mass scaling . . . . . . . . . . . . . . . . . . . . . . . . . 7

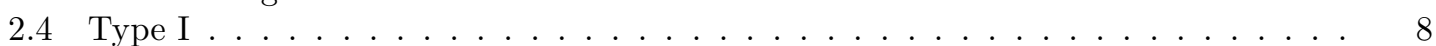

2.5 Coordinate choices for the dynamical systems picture . . . . . . . . . . . . 9 9

2.6 Approximate self-similarity and universality classes . . . . . . . . . . . . . . 9 9

2.7 The analogy with critical phase transitions . . . . . . . . . . . . . . 10

3 The scalar field 12

3.1 Field equations in spherical symmetry . . . . . . . . . . . . . . . 12

3.2 The black hole threshold . . . . . . . . . . . . . . . . . . . . 13

3.3 Global structure of the critical solution . . . . . . . . . . . . . . . . 14 
3.4 Near-critical spacetimes and naked singularities . . . . . . . . . . . . . . 15

3.5 Electric charge . . . . . . . . . . . . . . . . . . . . . . 17

3.6 Self-interaction potential . . . . . . . . . . . . . . . . . . . . 19

3.7 Nonspherical perturbations: stability and angular momentum . . . . . . . . . . . 20

4 More spherical symmetry $\quad 20$

4.1 Matter obeying wave equations ...................... 21

4.1.1 2d nonlinear $\sigma$ model . . . . . . . . . . . . . . . . . . . . . . . 21 21

4.1 .2 Spherical Einstein- $S U(2)$ sigma model . . . . . . . . . . . . . . . . . 22

4.1.3 Einstein-Yang-Mills ..................... . . . 22

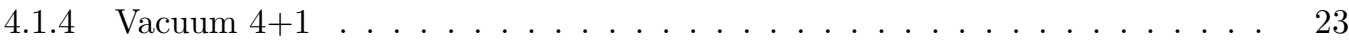

4.1.5 Scalar field collapse in $2+1$. . . . . . . . . . . . . . . . . . . . . . . . . . . . . . . . . . . . . . .

4.1.6 Scalar field collapse in higher dimensions . . . . . . . . . . . . . . . . . . 24

4.1.7 Other systems obeying wave equations . . . . . . . . . . . . . . . . 24

4.2 Perfect fluid matter . . . . . . . . . . . . . . . . . . . . . . 26

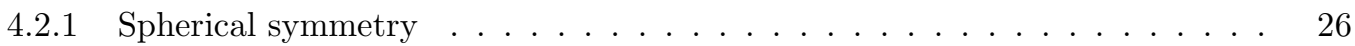

4.2.2 Nonspherical perturbations . . . . . . . . . . . . . . . . . . 27

4.3 Collisionless matter . . . . . . . . . . . . . . . . . . . . . 27

4.4 Criticality in singularity formation without gravitational collapse . . . . . . . . . 28

4.5 Analytic studies and toy models . . . . . . . . . . . . . . . . . . 29

4.5.1 Exact solutions of Einstein-Klein-Gordon . . . . . . . . . . . . . . . 29

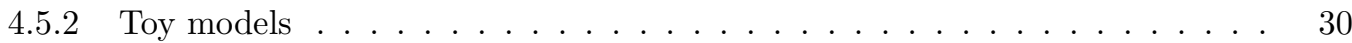

4.6 Quantum effects . . . . . . . . . . . . . . . . . . 30

5 Beyond spherical symmetry $\quad \mathbf{3 0}$

5.1 Perturbative approach to angular momentum . . . . . . . . . . . . . . 31

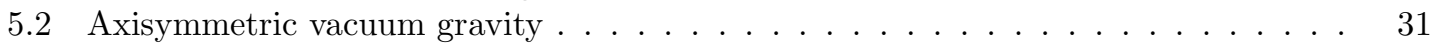

5.3 Scalar field . . . . . . . . . . . . . . . . . . . . . . 32

5.4 Neutron star collision in axisymmetry . . . . . . . . . . . . . . . . . . . . 34

5.5 Black hole collisions ........................... 34

\section{Introduction}

\subsection{Overview of the subject}

Take generic initial data in general relativity, adjust any one parameter $p$ of the initial data to the threshold of black hole formation, and compare the resulting spacetimes as a function of $p$. In many situations, the following critical phenomena are then observed:

- Near the threshold, black holes with arbitrarily small masses can be created, and the black hole mass scales as

$$
M \propto\left(p-p_{*}\right)^{\gamma},
$$

where $p$ parameterises the initial data and black holes form for $p>p_{*}$.

- The critical exponent $\gamma$ is universal with respect to initial data, that is, independent of the particular 1-parameter family, although it depends on the type of collapsing matter.

- In the region of large curvature before black hole formation, the spacetime approaches a self-similar solution which is also universal with respect to initial data, the critical solution. 
Critical phenomena were discovered by Choptuik [46] in numerical simulations of a spherical scalar field. They have been found in numerous other numerical and analytical studies in spherical symmetry, and a few in axisymmetry; in particular critical phenomena have been seen in the collapse of axisymmetric gravitational waves in vacuum [1. Scaling laws similar to the one for black hole mass have been discovered for black hole charge and conjectured for black hole angular momentum.

It is still unclear how universal critical phenomena in collapse are with respect to matter types and beyond spherical symmetry, in particular for vacuum collapse. Progress is likely to be made over the next few years with better numerical simulations.

Critical phenomena can usefully be described in dynamical systems terms. A critical solution is then characterised as an attracting fixed point within a surface that divides two basins of attraction, a critical surface in phase space. Such a fixed point can be either a stationary spacetime, or one that is scale-invariant and self-similar. The latter is relevant for the ("type II") critical phenomena sketched above. We shall also see how the dynamical systems approach establishes a connection with critical phenomena in statistical mechanics.

Therefore, we could define the field of critical phenomena in gravitational collapse as the study of the boundaries among the basins of attraction of different end states of self-gravitating systems, such as black hole formation or dispersion. In our view, the main physical motivation for this study is that those critical solutions which are self-similar provide a way of achieving arbitrarily large spacetime curvature outside a black hole, and in the limit a naked singularity, by fine-tuning generic initial data for generic matter to the black hole threshold. Those solutions are therefore likely to be important for quantum gravity and cosmic censorship.

\subsection{Plan of this review}

Faced with more material to review, we have attempted to make this update shorter and more systematic than the original 1999 version. We begin with the abstract theory in Sec. 2, Since 1999, progress on the theory side has mainly been made on the global spacetime structure of the critical solution and cosmic censorship in the spherical scalar field model. We have included this new material in an enlarged Sec. 3 on the spherical scalar field, although we hope it will turn out to be sufficiently generic to merit inclusion in Sec. 2, Nonspherical perturbations of the spherical scalar field are also discussed in Sec. B.

In Sec. 4 we review the rich phenomenology that has been found in many other systems restricted to spherical symmetry. Numerical work in spherical symmetry has proliferated since 1999, but we have tried to keep this section as short as possible. There has been less progress in going beyond spherical symmetry than we anticipated in 1999, even though we continue to believe that important results await there. What is known today is summarised in Sec. 5 .

The reader unfamiliar with the topic is advised to begin with either Secs. 2.1 2.3, which give the key theory of universality, self-similarity and scaling, or Secs. 3.1 3.2, which describe the classic example, the massless scalar field.

This review is limited to numerical and theoretical work on phenomena at the threshold of black hole formation in 3+1-dimensional general relativity. We report only briefly on work in higher and lower spacetime dimensions and non-gravity systems that may be relevant as toy models for general relativity. We exclude other work on self-similarity in general relativity and work on critical phenomena in other areas of physics.

Other reviews on the subject are [124, 13, [92, 96, [48, 49, 32, 98, 148. The 2002 review [101 by Gundlach gives more detailed explanations on some of the basic aspects of the theory. 


\section{Theory}

In this Section we describe the basic theory underlying critical collapse of the type that forms arbitrarily small black holes (later called type II, see also Sec.2.4). We begin with the mathematical origin of its three main characteristics, which were already summarised in the introduction:

- universality with respect to initial data;

- scale-invariance of the critical solution;

- black hole mass scaling.

\subsection{Universality}

Consider GR as an infinite-dimensional continuous dynamical system. Points in the phase space are initial data sets (3-metric, extrinsic curvature and suitable matter variables, which together obey the Einstein constraints). We evolve with the Einstein equations in a suitable gauge (see Sec. 2.5). Solution curves of the dynamical system are spacetimes obeying the Einstein-matter equations, sliced by specific Cauchy surfaces of constant time $t$.

An isolated system in GR can end up in qualitatively different stable end states. Two possibilities are the formation of a single black hole in collapse, or complete dispersion of the mass-energy to infinity. For a massless scalar field in spherical symmetry, these are the only possible end states (see Sec. 3). Any point in phase space can be classified as ending up in one or the other type of end state. The entire phase space therefore splits into two halves, separated by a "critical surface".

A phase space trajectory that starts on a critical surface by definition never leaves it. A critical surface is therefore a dynamical system in its own right, with one dimension fewer than the full system. If it has an attracting fixed point, such a point is called a critical point. It is an attractor of codimension one in the full system, and the critical surface is its attracting manifold. The fact that the critical solution is an attractor of codimension one is visible in its linear perturbations: it has an infinite number of decaying perturbation modes spanning the tangent plane to the critical surface, and a single growing mode not tangential to it.

As illustrated in Figs. 1 and 2, any trajectory beginning near the critical surface, but not necessarily near the critical point, moves almost parallel to the critical surface towards the critical point. Near the critical point the evolution slows down, and eventually moves away from the critical point in the direction of the growing mode. This is the origin of universality. All details of the initial data have been forgotten, except for the distance from the black hole threshold. The closer the initial phase point is to the critical surface, the more the solution curve approaches the critical point, and the longer it will remain close to it. We should stress that this phase picture is extremely simplified. Some of the problems associated with this simplification are discussed in Sec. 2.5.

\subsection{Self-similarity}

Fixed points of dynamical systems often have additional symmetries. In the case of type II critical phenomena, the critical point is a spacetime that is self-similar, or scale-invariant. These symmetries can be discrete or continuous. The critical solution of a spherically symmetric perfect fluid (see Sec. 4.2), has continuous self-similarity (CSS). A CSS spacetime is one that admits a homothetic vector field $\xi$, defined by [36]:

$$
\mathcal{L}_{\xi} g_{a b}=2 g_{a b} .
$$




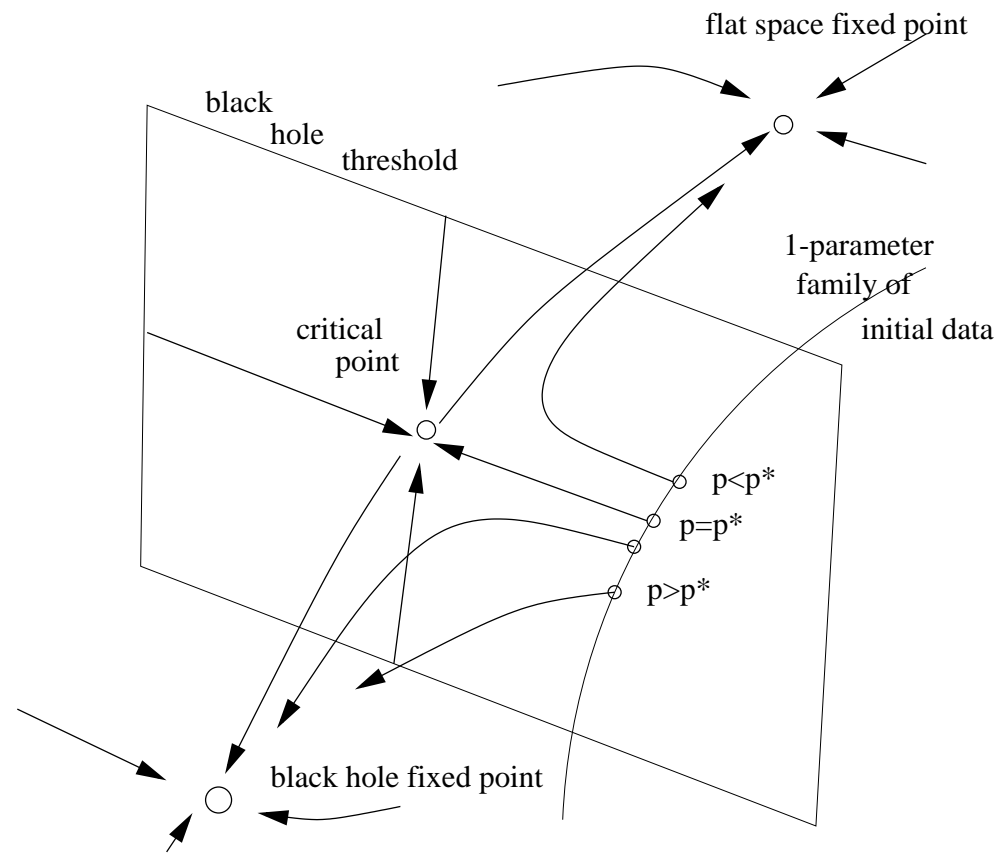

Figure 1: The phase space picture for the black hole threshold in the presence of a critical point. Every point correspond to an initial data set, that is, a 3-metric, extrinsic curvature, and matter fields. (In type II critical collapse these are only up to scale). The arrow lines are solution curves, corresponding to spacetimes, but the critical solution, which is stationary (type I) or self-similar (type II) is represented by a point. The line without an arrow is not a time evolution, but a 1parameter family of initial data that crosses the black hole threshold at $p=p_{*}$. The 2-dimensional plane represents an $(\infty-1)$-dimensional hypersurface, but the third dimension represents really only one dimension. 


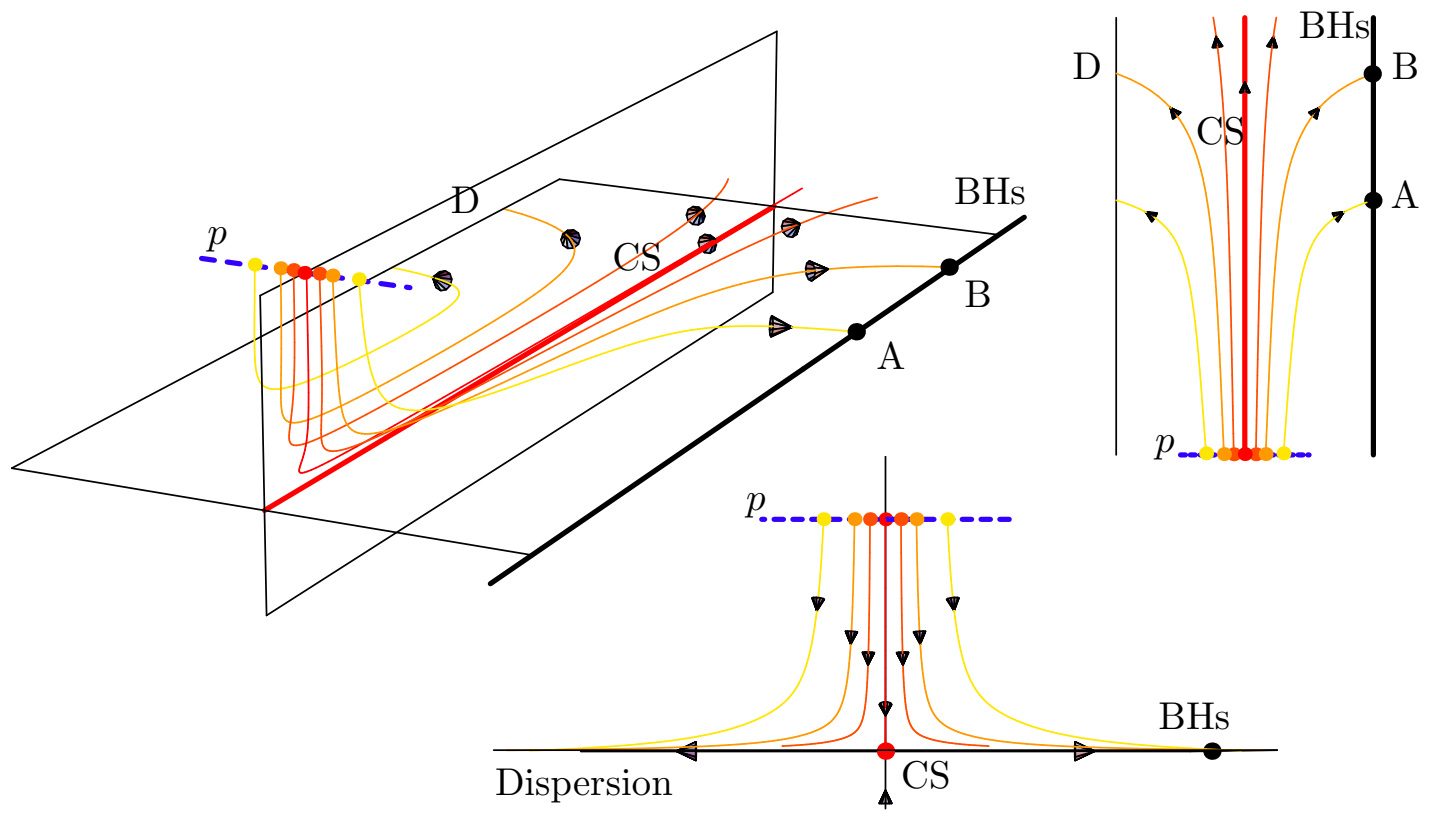

Figure 2: A different phase space picture, specifically for type II critical collapse, and two 2dimensional projections of the same picture. In contrast with Fig. 1, one dimension of the two representing the (infinitely many) decaying modes has been suppressed. The additional axis now represents a global scale which was suppressed in Fig. 1, so that the scale-invariant critical solution CS is now represented as a straight line (in red). Several members of a family of initial conditions (in blue) are attracted by the critical solution and then depart from it towards black hole formation (A or B) or dispersion (D). Perfectly fine-tuned initial data asymptote to the critical solution with decreasing scale. Initial conditions starting closer to perfect fine tuning produce smaller black holes, such that the parameter along the line of black hole endstates is $-\ln M_{B H}$. Two 2-dimensional projections of the same picture are also given. The horizontal projection of this figure is the same as the vertical projection of Fig. 1. 
In coordinates $x^{\mu}=\left(\tau, x^{i}\right)$ adapted to the symmetry, so that

$$
\xi=-\frac{\partial}{\partial \tau}
$$

the metric coefficients are of the form

$$
g_{\mu \nu}\left(\tau, x^{i}\right)=e^{-2 \tau} \tilde{g}_{\mu \nu}\left(x^{i}\right),
$$

where the coordinate $\tau$ is the negative logarithm of a spacetime scale, and the remaining three coordinates $x^{i}$ can be thought of angles around the singular spacetime point $\tau=\infty$ (see Sec. 3.3).

The critical solution of other systems, in particular the spherical scalar field (see Sec. 3i) and axisymmetric gravitational waves (see Sec. 5.2), show discrete self-similarity (DSS). The simplest way of defining DSS is in adapted coordinates, where

$$
g_{\mu \nu}\left(\tau, x^{i}\right)=e^{-2 \tau} \tilde{g}_{\mu \nu}\left(\tau, x^{i}\right),
$$

such that $g_{\mu \nu}\left(\tau, x^{i}\right)$ is periodic in $\tau$ with period $\Delta$. More formally, DSS can be defined as a discrete conformal isometry 91 .

Using the gauge freedom of general relativity, the lapse and shift in the ADM formalism can be chosen (non-uniquely) so that the coordinates become adapted coordinates if and when the solution becomes self-similar (see Sec. 2.5). $\tau$ is then both a time coordinate (in the usual sense that surfaces of constant time are Cauchy surfaces), and the logarithm of overall scale at constant $x^{i}$. The minus sign in (3) and hence (4) and (5), is a convention assuming that smaller scales are in the future. The time parameter used in Fig. 2 is of this type.

\subsection{Mass scaling}

Let $Z$ stand for a set of scale-invariant variables of the problem, such as $\tilde{g}_{\mu \nu}$ and suitably rescaled matter variables. If the dynamics is scale-invariant (this is the case exactly for example for the scalar field, and approximately for other systems, see Sec. 2.6), then $Z(x)$ is an element of the phase space factored by overall scale, and $Z(x, \tau)$ a solution. Note that $Z(x)$ is an initial data set for GR only up to scale. The overall scale is supplied by $\tau$.

For simplicity, assume that the critical solution is CSS. It can then be written as $Z(x, \tau)=$ $Z_{*}(x)$. Its linear perturbations can depend on $\tau$ only exponentially. To linear order, the solution near the critical point must be of the form

$$
Z(x, \tau) \simeq Z_{*}(x)+\sum_{i=1}^{\infty} C_{i}(p) e^{\lambda_{i} \tau} Z_{i}(x) .
$$

The perturbation amplitudes $C_{i}$ depend on the initial data, and hence on $p$. As $Z_{*}$ is a critical solution, by definition there is exactly one $\lambda_{i}$ with positive real part (in fact it is purely real), say $\lambda_{0}$. As $\tau \rightarrow \infty$, all other perturbations vanish. In the following we consider this limit, and retain only the one growing perturbation.

From our phase space picture, the evolution ends at the critical solution for $p=p_{*}$, so we must have $C_{0}\left(p_{*}\right)=0$. Linearising in $p$ around $p_{*}$, we obtain

$$
\lim _{\tau \rightarrow \infty} Z(x, \tau) \simeq Z_{*}(x)+\frac{d C_{0}}{d p}\left(p-p_{*}\right) e^{\lambda_{0} \tau} Z_{0}(x) .
$$

For $p \neq p_{*}$, but close to it, the solution has the approximate form (7) over a range of $\tau$. Now we extract Cauchy data at one particular $p$-dependent value of $\tau$ within that range, namely $\tau_{*}$ defined by

$$
\frac{d C_{0}}{d p}\left(p-p_{*}\right) e^{-\lambda_{0} \tau_{*}} \equiv \epsilon,
$$


where $\epsilon$ is some constant $\ll 1$ such that at this $\tau$ the linear approximation is still valid. At sufficiently large $\tau$, the linear perturbation has grown so much that the linear approximation breaks down, and for $C_{0}>0$ a black hole forms while for $C_{0}<0$ the solution disperses. The crucial point is that we need not follow this evolution in detail, nor does the precise value of $\epsilon$ matter. It is sufficient to note that the Cauchy data at $\tau=\tau_{*}$ are

$$
Z\left(x, \tau_{*}\right) \simeq Z_{*}(x)+\epsilon Z_{0}(x) .
$$

Due to the funnelling effect of the critical solution, the data at $\tau_{*}$ is always the same, except for an overall scale, which is given by $e^{-\tau_{*}}$. For example, the physical spacetime metric, with dimension (length) $)^{2}$ is given by $g_{\mu \nu}=e^{-2 \tau} \tilde{g}_{\mu \nu}$, and similar scalings hold for the matter variables according to their dimension. In particular, as $e^{-\tau_{*}}$ is the only scale in the initial data (9), the mass of the final black hole must be proportional to that scale. Therefore

$$
M \propto e^{-\tau_{*}} \propto\left(p-p_{*}\right)^{1 / \lambda_{0}},
$$

and, comparing with Eq. (1), we have found the critical exponent $\gamma=1 / \lambda_{0}$.

When the critical solution is DSS, a periodic or fine structure of small amplitude is superimposed on this basic power law 94,122 :

$$
\ln M=\gamma \ln \left(p-p_{*}\right)+c+f\left(\gamma \ln \left(p-p_{*}\right)+c\right),
$$

where $f(z)$ has period $\Delta$ and is universal, and only $c$ depends on the initial data. As the critical solution is periodic in $\tau$ with period $\Delta$ the number $N$ of scaling "echos" is approximated by

$$
N \simeq \Delta^{-1} \gamma \ln \left|p-p_{*}\right|+\text { const. }
$$

Note that this holds for both supercritical and subcritical solutions.

\subsection{Type I}

In type I critical phenomena, the same phase space picture as in Sec. 2.1 applies, but the critical solution is now stationary or time-periodic instead of self-similar or scale-periodic. It also has a finite mass and can be thought of as a metastable star. (Type I and II were so named after first and second order phase transitions in statistical mechanics, in which the order parameter is discontinuous and continuous, respectively.) Universality in this context implies that the black hole mass near the threshold is independent of the initial data, namely a certain fraction of the mass of the stationary critical solution. The dimensionful quantity that scales is not the black hole mass, but the lifetime $t_{p}$ of the intermediate state where the solution is approximated by the critical solution. This is clearly

$$
t_{p}=-\frac{1}{\lambda_{0}} \ln \left|p-p_{*}\right|+\text { const. }
$$

Type I critical phenomena occur when a mass scale in the field equations becomes dynamically relevant. (This scale does not necessarily set the mass of the critical solution absolutely: there could be a family of critical solutions selected by the initial conditions.) Conversely, as the type II power law is scale-invariant, type II phenomena occur in situations where either the field equations do not contain a scale, or this scale is dynamically irrelevant. Many systems, such as the massive scalar field, show both type I and type II critical phenomena, in different regions of the space of initial data [33]. 


\subsection{Coordinate choices for the dynamical systems picture}

The time evolution of Cauchy data in GR can only be considered as a dynamical system if the ADM evolution equations are complemented by a prescription for the lapse and shift. To realise the phase space picture of Sec. 2.1, the critical solution must be a fixed point or limit cycle. We have seen how coordinates adapted to the self-similarity can be constructed, but is there a prescription of the lapse and shift for arbitrary initial data, such that, given initial data for the critical solution, the resulting time evolution actively drives the metric to a form (5) that explicitly displays the self-similarity?

Garfinkle and Gundlach 82 have suggested several combinations of lapse and shift conditions that leave CSS spacetimes invariant and turn the Choptuik DSS spacetime into a limit cycle. (See [87, 78. for partial successes.) Among these, the combination of maximal slicing with minimal strain shift has been suggested in a different context but for related reasons [176]. Maximal slicing requires the initial data slice to be maximal $\left(K_{a}{ }^{a}=0\right)$, but other prescriptions, such as freezing the trace of $K$ together with minimal distortion, allow for an arbitrary initial slice with arbitrary spatial coordinates.

All these coordinate conditions are elliptic equations that require boundary conditions, and will turn CSS spacetimes into fixed points (or DSS into limit cycles) only given correct boundary conditions. Roughly speaking, these boundary conditions require a guess of how far the slice is from the accumulation point $t=t_{*}$, and answers to this problem only exist in spherical symmetry. Appropriate boundary conditions are also needed if the dynamical system is extended to include the lapse and shift as evolved variables, turning the elliptic equations for the lapse and shift into hyperbolic or parabolic equations.

Turning a CSS or stationary spacetime into a fixed point of the dynamical system also requires an appropriate choice of the phase space variables $Z\left(x^{i}\right)$. To capture CSS (or DSS) solutions, one needs scale-invariant variables. Essentially, these can be constructed by dimensional analysis. The coordinates $x^{i}$ and $\tau$ are dimensionless, $l e^{-\tau}$ has dimension length, and $g_{\mu \nu}$ has dimension $l^{2}$. The scaling for the ADM and any matter variables follows.

Even with a prescription for the lapse and shift in place, a given spacetime does not correspond to a unique trajectory in phase space. Rather, for each initial slice through the same spacetime one obtains a different slicing of the entire spacetime. A possibility for avoiding this ambiguity would be to restrict the phase space further, for example by restricting possible data sets to maximal or constant extrinsic curvature slices.

Another open problem is that in order to talk about attractors and repellers on the phase space we need to define a norm on a suitable function space which includes both asymptotically flat data and data for the exact critical solution. The norm itself must favour the central region and ignore what is further out and asymptotically flat if all black holes of the same mass are to be considered as the same endstate.

\subsection{Approximate self-similarity and universality classes}

The field equations for the massless scalar field coupled to the Einstein equations are scale-free. Realistic matter models introduce length scales, and the field equations then do not allow for exactly self-similar solutions. They may however admit solutions which are CSS or DSS asymptotically on small spacetime scales as the dimensionful parameters become irrelevant, including type II critical solutions [47, 33, 50. This can be explored by a formal expansion in powers of the small parameter $L e^{-\tau}$, where $L$ is a parameter with dimensions length in the evolution equations. The zeroth order of the expansion is the self-similar critical solution of the system with $L=0$. A similar ansatz can be made for the linear perturbations of the resulting background. The zeroth order of the background expansion determines $\Delta$ exactly and independently of $L$, and the zeroth order term of the linear perturbation expansion determines the critical exponent $1 / \lambda_{0}$ exactly, so that there 
is no need in practice to calculate any higher orders in $L$ to make predictions for type II critical phenomena where they occur. (With $L \neq 0$, the basin of attraction of the type II critical solution will depend on $L$, and type I critical phenomena may also occur, see Sec. 2.4. A priori, there could also be more than one type II critical solution for $L=0$, although this has not been observed.)

This procedure has been carried out for the Einstein-Yang-Mills system 93] and for massless scalar electrodynamics 102. Both systems have a single length scale $1 / e$ (in geometric units $c=G=1$ ), where $e$ is the gauge coupling constant. All values of $e$ can be said to form one universality class of field equations 108 represented by $e=0$. This notion of universality classes is fundamentally the same as in statistical mechanics. Other examples include modifications to the perfect fluid equation of state that do not affect the limit of high density 152. A simple example is that any scalar field potential $V(\phi)$ becomes dynamically irrelevant compared to the kinetic energy $|\nabla \phi|^{2}$ in a self-similar solution [47, so that all scalar fields with potentials are in the universality class of the free massless scalar field. Surprisingly, even two different models like the $S U(2)$ Yang-Mills and $S U(2)$ Skyrme models in spherical symmetry are members of the same universality class 20 .

If there are several scales $L_{0}, L_{1}, L_{2}$ etc. present in the problem, a possible approach is to set the arbitrary scale in (28) equal to one of them, say $L_{0}$, and define the dimensionless constants $l_{i}=L_{i} / L_{0}$ from the others. The scope of the universality classes depends on where the $l_{i}$ appear in the field equations. If a particular $L_{i}$ appears in the field equations only in positive integer powers, the corresponding $l_{i}$ appears only multiplied by $e^{-\tau}$, and will be irrelevant in the scaling limit. All values of this $l_{i}$ therefore belong to the same universality class. From the example above, adding a quartic self-interaction $\lambda \phi^{4}$ to the massive scalar field gives rise to the dimensionless number $\lambda / \mathrm{m}^{2}$ but its value is an irrelevant (in the language of renormalisation group theory) parameter.

Contrary to the statement in 102, we conjecture that massive scalar electrodynamics, for any values of $e$ and $m$, is in the universality class of the massless uncharged scalar field in a region of phase space where type II critical phenomena occur. Examples of dimensionless parameters which do change the universality class are the $k$ of the perfect fluid, the $\kappa$ of the 2-dimensional sigma model or, probably, a conformal coupling of the scalar field [45] (the numerical evidence is weak but a dependence should be expected).

\subsection{The analogy with critical phase transitions}

Some basic aspects of critical phenomena in gravitational collapse, such as fine-tuning, universality, scale-invariant physics, and critical exponents for dimensionful quantities, can also be identified in critical phase transitions in statistical mechanics (see [193] for an introductory textbook).

From an abstract point of view, the objective of statistical mechanics is to derive relations between macroscopic observables $A$ of the system and macroscopic external forces $f$ acting on it, by considering ensembles of microscopic states of the system. The expectation values $\langle A\rangle$ can be generated as partial derivatives of the partition function

$$
Z(\mu, f)=\sum_{\text {microstates }} e^{-H(\text { microstate }, \mu, f)}
$$

Here the $\mu$ are parameters of the Hamiltonian such as the strength of intermolecular forces, and $f$ are macroscopic quantities which are being controlled, such as the temperature or magnetic field.

Phase transitions in thermodynamics are thresholds in the space of external forces $f$ at which the macroscopic observables $A$, or one of their derivatives, change discontinuously. We consider two examples: the liquid-gas transition in a fluid, and the ferromagnetic phase transition.

The liquid-gas phase transition in a fluid occurs at the boiling curve $p=p_{b}(T)$. In crossing this curve, the fluid density changes discontinuously. However, with increasing temperature, the 
difference between the liquid and gas density on the boiling curve decreases, and at the the critical point $\left(p_{*}=p_{b}\left(T_{*}\right), T_{*}\right)$ it vanishes as a non-integer power:

$$
\rho_{\text {liquid }}-\rho_{\text {gas }} \sim\left(T_{*}-T\right)^{\gamma} .
$$

At the critical point an otherwise clear fluid becomes opaque, due to density fluctuations appearing on all scales up to scales much larger than the underlying atomic scale, and including the wavelength of light. This indicates that the fluid near its critical point is approximately scale-invariant (for some range of scales between the size of molecules and the size of the container).

In a ferromagnetic material at high temperatures, the magnetisation $\mathbf{m}$ of the material (alignment of atomic spins) is determined by the external magnetic field $\mathbf{B}$. At low temperatures, the material shows a spontaneous magnetisation even at zero external field. In the absence of an external field this breaks rotational symmetry: the system makes a random choice of direction. With increasing temperature, the spontaneous magnetisation $\mathbf{m}$ decreases and vanishes at the Curie temperature $T_{*}$ as

$$
|\mathbf{m}| \sim\left(T_{*}-T\right)^{\gamma} .
$$

Again, the correlation length, or length scale of a typical fluctuation, diverges at the critical point, indicating scale-invariant physics.

Quantities such as $|\mathbf{m}|$ or $\rho_{\text {liquid }}-\rho_{\text {gas }}$ are called order parameters. In statistical mechanics, one distinguishes between first-order phase transitions, where the order parameter changes discontinuously, and second-order, or critical, ones, where it goes to zero continuously. One should think of a critical phase transition as the critical point where a line of first-order phase transitions ends as the order parameter vanishes. This is already clear in the fluid example. In the ferromagnet example, at first one seems to have only the one parameter $T$ to adjust. But in the presence of a very weak external field, the spontaneous magnetisation aligns itself with the external field $\mathbf{B}$, while its strength is to leading order independent of $\mathbf{B}$. The function $\mathbf{m}(\mathbf{B}, T)$ therefore changes discontinuously at $\mathbf{B}=0$. The line $\mathbf{B}=0$ for $T<T_{*}$ is therefore a line of first order phase transitions between directions (if we consider one spatial dimension only, between $\mathbf{m}$ up and $\mathbf{m}$ down). This line ends at the critical point $\left(\mathbf{B}=0, T=T_{*}\right)$ where the order parameter $|\mathbf{m}|$ vanishes. The critical value $\mathbf{B}=0$ of $\mathbf{B}$ is determined by symmetry; by contrast $p_{*}$ depends on microscopic properties of the material.

We have already stated that a critical phase transition involves scale-invariant physics. In particular, the atomic scale, and any dimensionful parameters associated with that scale, must become irrelevant at the critical point. This is taken as the starting point for obtaining properties of the system at the critical point.

One first defines a semi-group acting on micro-states: the renormalisation group. Its action is to group together a small number of adjacent particles as a single particle of a fictitious new system by using some averaging procedure. This can also be done in a more abstract way in Fourier space. One then defines a dual action of the renormalisation group on the space of Hamiltonians by demanding that the partition function is invariant under the renormalisation group action:

$$
\sum_{\text {microstates }} e^{-H}=\sum_{\text {microstates' }^{\prime}} e^{-H^{\prime}} .
$$

The renormalised Hamiltonian is in general more complicated than the original one, but it can be approximated by the same Hamiltonian with new values of the parameters $\mu$ and external forces $f$. (At this stage it is common to drop the distinction between $\mu$ and $f$, as the new $\mu^{\prime}$ and $f^{\prime}$ depend on both $\mu$ and $f$.) Fixed points of the renormalisation group correspond to Hamiltonians with the parameters at their critical values. The critical values of many of these parameters will be 0 or $\infty$, meaning that the dimensionful parameters they were originally associated with are irrelevant. 
Because a fixed point of the renormalisation group can not have a preferred length scale, the only parameters that can have nontrivial values are dimensionless.

The behaviour of thermodynamical quantities at the critical point is in general not trivial to calculate. But the action of the renormalisation group on length scales is given by its definition. The blowup of the correlation length $\xi$ at the critical point is therefore the easiest critical exponent to calculate. The same is true for the black hole mass, which is just a length scale. We can immediately reinterpret the mathematics of Section 2.3 as a calculation of the critical exponent for $\xi$, by substituting the correlation length $\xi$ for the black hole mass $M, T_{*}-T$ for $p-p_{*}$, and taking into account that the $\tau$-evolution in critical collapse is towards smaller scales, while the renormalisation group flow goes towards larger scales: $\xi$ therefore diverges at the critical point, while $M$ vanishes.

In type II critical phenomena in gravitational collapse, we should think of the black hole mass as being controlled by the functions $P$ and $Q$ on phase space defined by Eq. (26). Clearly, $P$ is the equivalent of the reduced temperature $T-T_{*}$. Gundlach [100] has suggested that the angular momentum of the initial data can play the role of $\mathbf{B}$, and the final black hole angular momentum the role of $\mathbf{m}$. Like the magnetic field, angular momentum is a vector, with a critical value that must be zero because all other values break rotational symmetry.

\section{The scalar field}

Critical phenomena in gravitational collapse were first discovered by Choptuik 45, 46, 47, in the model of a spherically symmetric, massless scalar field $\phi$ minimally coupled to general relativity. The scalar field matter is both simple, and acts as a toy model in spherical symmetry for the effects of gravitational radiation. Given that it is still the best-studied model in spherical symmetry, we review it here as a case study. For other numerical work on this model, see [105, 77, 107, 174, 171. Important analytical studies of gravitational collapse in this model have been carried out by Christodoulou [55, 56, 57, 58, 59, 60, 61].

We first review the field equations and Choptuik's observations at the black hole threshold, mainly as a concrete example for the general ideas discussed above. We then summarise more recent work on the global structure of Choptuik's critical solution, which throws an interesting light on cosmic censorship. In particular, the exact critical solution contains a curvature singularity that is locally and globally naked, and any critical solution obtained in the limit of perfect fine-tuning of asymptotically flat initial data is at least locally naked. By perturbing around spherical symmetry, the stability of the Choptuik solution in the full phase space can be investigated, and the scaling of black hole angular momentum can be predicted. By embedding the real scalar field in scalar electrodynamics and perturbing around the Choptuik solution, the scaling of black hole charge can be predicted.

\subsection{Field equations in spherical symmetry}

The Einstein equations are

$$
G_{a b}=8 \pi\left(\nabla_{a} \phi \nabla_{b} \phi-\frac{1}{2} g_{a b} \nabla_{c} \phi \nabla^{c} \phi\right),
$$

and the matter equation is

$$
\nabla_{a} \nabla^{a} \phi=0 .
$$

Note that the matter equation of motion is contained within the contracted Bianchi identities. Choptuik chose Schwarzschild-like coordinates

$$
d s^{2}=-\alpha^{2}(r, t) d t^{2}+a^{2}(r, t) d r^{2}+r^{2} d \Omega^{2},
$$


where $d \Omega^{2}=d \theta^{2}+\sin ^{2} \theta d \varphi^{2}$ is the metric on the unit 2-sphere. This choice of coordinates is defined by the radius $r$ giving the surface area of 2 -spheres as $4 \pi r^{2}$, and by $t$ being orthogonal to $r$ (polar-radial coordinates). One more condition is required to fix the coordinate completely. Choptuik chose $\alpha=1$ at $r=0$, so that $t$ is the proper time of the central observer.

In the auxiliary variables

$$
\Phi=\phi_{, r}, \quad \Pi=\frac{a}{\alpha} \phi_{, t},
$$

the wave equation becomes a first-order system,

$$
\begin{aligned}
\Phi_{, t} & =\left(\frac{\alpha}{a} \Pi\right)_{, r}, \\
\Pi_{, t} & =\frac{1}{r^{2}}\left(r^{2} \frac{\alpha}{a} \Phi\right)_{, r} .
\end{aligned}
$$

In spherical symmetry there are four algebraically independent components of the Einstein equations. Of these, one is a linear combination of derivatives of the other and can be disregarded. The other three contain only first derivatives of the metric, namely $a_{, t}, a_{, r}$ and $\alpha_{, r}$, and are

$$
\begin{aligned}
\frac{a_{, r}}{a}+\frac{a^{2}-1}{2 r} & =2 \pi r\left(\Pi^{2}+\Phi^{2}\right), \\
\frac{\alpha_{, r}}{\alpha}-\frac{a_{, r}}{a}-\frac{a^{2}-1}{r} & =0, \\
\frac{a_{, t}}{\alpha} & =4 \pi r \Phi \Pi .
\end{aligned}
$$

Because of spherical symmetry, the only dynamics is in the scalar field equations (22). The metric can be found by integrating the ODEs (23) and (24) for $a$ and $\alpha$ at any fixed $t$, given $\phi$ and $\Pi$. Eq. (25) can be ignored in this "fully constrained" evolution scheme.

\section{$3.2 \quad$ The black hole threshold}

The free data for the system are the two functions $\Pi(r, 0)$ and $\Phi(r, 0)$. Choptuik investigated several one-parameter families of such data by evolving the data for many different values of the parameter. Simple examples of such families are $\Pi(r, 0)=0$ and a Gaussian for $\Phi(r, 0)$, with the parameter $p$ taken to be either the amplitude of the Gaussian, with the width and centre fixed, or the width, with position and amplitude fixed, or the position, with width and amplitude fixed. For sufficiently small amplitude (or the peak sufficiently wide), the scalar field will disperse, and for sufficiently large amplitude it will form a black hole.

Generic one-parameter families behave in this way, but this is difficult to prove in generality. Christodoulou showed for the spherically symmetric scalar field system that data sufficiently weak in a well-defined way evolve to a Minkowski-like spacetime [56, 59, and that a class of sufficiently strong data forms a black hole [58.

Choptuik found that in all 1-parameter families of initial data he investigated he could make arbitrarily small black holes by fine-tuning the parameter $p$ close to the black hole threshold. An important fact is that there is nothing visibly special to the black hole threshold. One cannot tell that one given data set will form a black hole and another one infinitesimally close will not, short of evolving both for a sufficiently long time.

As $p \rightarrow p_{*}$ along the family, the spacetime varies on ever smaller scales. Choptuik developed numerical techniques that recursively refine the numerical grid in spacetime regions where details arise on scales too small to be resolved properly. In the end, he could determine $p_{*}$ up to a relative precision of $10^{-15}$, and make black holes as small as $10^{-6}$ times the ADM mass of the 
spacetime. The power-law scaling (10) was obeyed from those smallest masses up to black hole masses of, for some families, 0.9 of the ADM mass, that is, over six orders of magnitude 47 . There were no families of initial data which did not show the universal critical solution and critical exponent. Choptuik therefore conjectured that $\gamma$ is the same for all one-parameter families of smooth, asymptotically flat initial data that depend smoothly on the parameter, and that the approximate scaling law holds ever better for arbitrarily small $p-p_{*}$.

It is an empirical fact that typical one-parameter families cross the threshold only once, so that there is every indication that it is a smooth submanifold, as we assumed in the phase space picture. Taking into account the discussion of mass scaling above, we can formally write the black hole mass as a functional of the initial data $z=(\phi(r, 0), \Pi(r, 0))$ exactly as

$$
M[z]=Q[z] H(P[z])(P[z])^{\gamma},
$$

where $P$ and $Q$ are smooth functions on phase space and $H$ is the Heaviside function. ( $Q$ could be absorbed into $P$ ).

In hindsight, polar-radial gauge is well-adapted to self-similarity. In this gauge, discrete selfsimilarity corresponds to

$$
Z(r, t)=Z\left(e^{n \Delta} r, e^{n \Delta} t\right)
$$

for any integer $n$, where $Z$ stands for any one of the dimensionless quantities $a, \alpha$ or $\phi$ (and therefore also for $r \Pi$ or $r \Phi)$. With

$$
x=-\frac{r}{t-t_{*}}, \quad \tau=-\ln \left(-\frac{t-t_{*}}{L}\right), \quad t<t_{*},
$$

discrete self-similarity is

$$
Z(x, \tau+\Delta)=Z(x, \tau) .
$$

The dimensionful constants $t_{*}$ and $L$ depend on the particular one-parameter family of solutions, but the dimensionless critical fields $a_{*}, \alpha_{*}$ and $\phi_{*}$, and in particular their dimensionless period $\Delta$, are universal. Empirically, $\Delta \simeq 3.44$ for the scalar field in numerical time evolutions, and $\Delta=3.445452402(3)$ from a numerical construction of the critical solution based on exact selfsimilarity and analyticity [149].

\subsection{Global structure of the critical solution}

In adapted coordinates, the metric of the critical spacetime is of the form $e^{-2 \tau}$ times a regular metric. From this general form alone, one can conclude that $\tau=\infty$ is a curvature singularity, where Riemann and Ricci invariants blow up like $e^{4 \tau}$ (unless the spacetime is flat), and which is at finite proper time from regular points in its past. The Weyl tensor with index position $C^{a} b c d$ is conformally invariant, so that components with this index position remain finite as $\tau \rightarrow \infty$. This type of singularity is called "conformally compactifiable" [184 or "isotropic" 89. For a classification of all possible global structures of spherically symmetric self-similar spacetimes see 103 .

The global structure of the scalar field critical solution was determined accurately in 149, by assuming analyticity at the centre of spherical symmetry and at the past light cone of the singularity (the self-similarity horizon, or SSH). The critical solution is then analytic up to the future lightcone of the singularity (the Cauchy horizon, or $\mathrm{CH}$ ). Global adapted coordinates $x$ and $\tau$ can be chosen so that the regular centre $r=0$, the SSH and the $\mathrm{CH}$ are all lines of constant $x$, and surfaces of constant $\tau$ are never tangent to $x$ lines. (A global $\tau$ is no longer a global time coordinate). This is illustrated in Fig. 4. 
Approaching the Cauchy horizon, the scalar field oscillates an infinite number of times but with the amplitude of the oscillations decaying to zero. The scalar field in regular adapted coordinates $(x, \tau)$ is of the form

$$
\phi(x, \tau) \simeq F_{\mathrm{reg}}(\tau)+|x|^{\epsilon} F_{\operatorname{sing}}[\tau+H(\tau)+K \ln |x|],
$$

where $F_{\text {reg }}(\tau), F_{\text {sing }}(\tau)$ and $H(\tau)$ are periodic with period $\Delta$, and the SSH is at $x=0$. These functions have been computed numerically to high accuracy, together with the constants $K$ and $\epsilon$. The scalar field itself is smooth with respect to $\tau$, and as $\epsilon>0$, it is continuous but not differentiable with respect to $x$ on the $\mathrm{CH}$ itself. The same is true for the metric and the curvature. Surprisingly, the ratio $m / r$ of the Hawking mass over the area radius on the Cauchy horizon is of order $10^{-6}$ but not zero (the value is known to eight significant figures).

As the $\mathrm{CH}$ itself is regular with smooth null data except for the singular point at its base, it is not intuitively clear why the continuation is not unique. A partial explanation is given in [149, where all $D S S$ continuations are considered. Within a DSS ansatz, the solution just to the future of the $\mathrm{CH}$ has the same form as (30). $F_{\text {reg }}(\tau)$ is the same on both sides, but $F_{\text {sing }}(\tau)$ can be chosen freely on the future side of the $\mathrm{CH}$. Within the restriction to DSS this function can be taken to parameterise the information that comes out of the naked singularity.

There is precisely one choice of $F_{\text {sing }}(\tau)$ on the future side that gives a regular centre to the future of the $\mathrm{CH}$, with the exception of the naked singularity itself, which is then a point. This continuation was calculated numerically, and is almost but not quite Minkowski in the sense that $m / r$ remains small everywhere to the future of the SSH.

All other DSS continuations have a naked, timelike central curvature singularity with negative mass. More exotic continuations including further Cauchy horizons would be allowed kinematically [40] but are not achieved dynamically if we assume that the continuation is DSS. The spacetime diagram of the generic DSS continued solution is given in Fig. 3 .

\subsection{Near-critical spacetimes and naked singularities}

Choptuik's results have an obvious bearing on the issue of cosmic censorship. (See [189] for a general review of cosmic censorship.) Roughly speaking, fine-tuning to the black hole threshold provides a set of data which is codimension one in the space of generic, smooth, asymptotically flat initial data, and whose time evolution contains at least the point singularity of the critical solution. The cosmic censorship hypothesis must therefore be formulated as "generic smooth initial data for reasonable matter do not form naked singularities". Here we look at the relation between fine-tuning and naked singularities in more detail.

Christodoulou 61 proves rigorously that naked singularity formation is not generic, but in a rather larger function space, functions of bounded variation, than one would naturally consider. In particular, the instability of the naked singularity found by Christodoulou is not differentiable on the past light cone. This is unnatural in the context of critical collapse, where the naked singularity can arise from generic (up to fine-tuning) smooth initial data, and the intersection of the past light cone of the singularity with the initial data surface is as smooth as the initial data elsewhere. It is therefore not clear how this theorem relates to the numerical and analytical results strongly indicating that naked singularities are codimension-1 generic within the space of smooth initial data.

First, consider the exact critical solution. The lapse $\alpha$ defined by (20) is bounded above and below in the critical solution. Therefore the redshift measured between constant $r$ observers located at any two points on an outgoing radial null geodesic in the critical spacetime to the past of the $\mathrm{CH}$ is bounded above and below. Within the exact critical solution, a point with arbitrarily high curvature can therefore be observed from a point with arbitrarily low curvature. Next consider a 


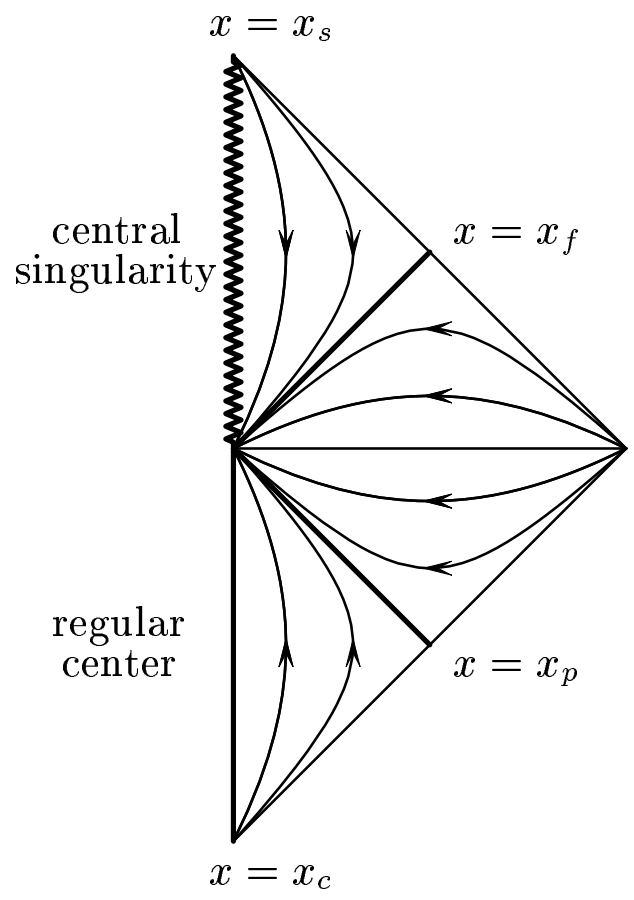

Figure 3: The spacetime diagram of all generic DSS continuations of the scalar field critical solution, from [149. The naked singularity is timelike, central, strong, and has negative mass. There is also a unique continuation where the singularity is replaced by a regular centre except at the spacetime point at the base of the $\mathrm{CH}$, which is still a strong curvature singularity. No other spacetime diagram is possible if the continuation is DSS. The lines with arrows are lines of constant adapted coordinate $x$, with the arrow indicating the direction of $\partial / \partial \tau$ towards larger curvature. 
spacetime where the critical solution in a central region is smoothly matched to an asymptotically flat outer region such that the resulting asymptotically flat spacetime contains a part of the critical solution that includes the singularity and a part of the $\mathrm{CH}$. In this spacetime, a point of arbitrarily large curvature can be seen from $\mathscr{I}^{+}$with finite redshift. This is illustrated in Fig. (4.

Now consider the evolution of asymptotically flat initial data that have been fine-tuned to the black hole threshold. The global structure of such spacetimes has been investigated numerically in [107, 74, 77, 171. Empirically, these spacetimes can be approximated near the singularity by (6). Almost all perturbations decay as the singularity is approached and the approximation becomes better, until the one growing perturbation (which by the assumption of fine-tuning starts out small) becomes significant. A maximal value of the curvature is then reached which is still visible from $\mathscr{I}^{+}$and which scales as 81

$$
R_{\max } \propto\left(p-p_{*}\right)^{2 \gamma} .
$$

Finally, consider the limit of perfect fine-tuning. The growing mode is then absent, and all other modes decay as the naked singularity is approached. Note that (6) suggests this is true regardless of the direction (future, past, or spacelike, depending on the value of $x$ ) in which the singularity is approached. This seems to be in conflict with causality: the issue is sensitively connected to the completeness of the modes $Z_{i}$ and to the stability of the Cauchy horizon and requires more investigation.

A complication in the supercritical case has been pointed out in 171. In the literature on supercritical evolutions, what is quoted as the black hole mass is in fact the mass of the first apparent horizon $(\mathrm{AH})$ that appears in the time slicing used by the code (spacelike or null). The black hole mass can and generically will be larger than the AH mass when it is first measured because of matter falling in later, and the region of maximal curvature may well be inside the event horizon, and hidden from observers at $\mathscr{I}^{+}$. (See Fig. 5 for an illustration). The true black hole mass can only be measured at $\mathscr{I}^{+}$, where it is defined to be the limit of the Bondi mass $m_{\mathrm{B}}$ as the Bondi time $u_{\mathrm{B}} \rightarrow \infty$. This was implemented in 171. Only one family of initial data was investigated, but in this family it was found that $m_{\mathrm{B}}$ converges to $10^{-4}$ of the initial Bondi mass in the fine tuning limit. More numerical evidence would be helpful, but the result is plausible. As the underlying physics is perfectly scale-invariant in the massless scalar field model, the minimum mass must be determined by the family of initial data through the infall of matter into the black hole. Simulations of critical collapse of a perfect fluid in a cosmological context show a similar lower bound 114 due to matter falling back after shock formation, but this may not be true for all initial data 151 .

\subsection{Electric charge}

Given the scaling power law for the black hole mass in critical collapse, one would like to know what happens if one takes a generic 1-parameter family of initial data with both electric charge and angular momentum (for suitable matter), and fine-tunes the parameter $p$ to the black hole threshold. A simple model for charged matter is a complex scalar field coupled to electromagnetism with the substitution $\nabla_{a} \rightarrow \nabla_{a}+i e A_{a}$, or scalar electrodynamics. (Note that in geometric units, black hole charge $Q$ has dimension of length, but the charge parameter $e$ has dimension 1/length.)

Gundlach and Martín-García [102] have studied scalar massless electrodynamics in spherical symmetry perturbatively. Clearly, the real scalar field critical solution of Choptuik is a solution of this system too. In fact, it remains a critical solution within massless scalar electrodynamics in the sense that it still has only one growing perturbation mode within the enlarged solution space. Some of its perturbations carry electric charge, but as they are all decaying, electric charge is a subdominant effect. The charge of the black hole in the critical limit is dominated by the most slowly decaying of the charged modes. From this analysis, a universal power-law scaling of the 


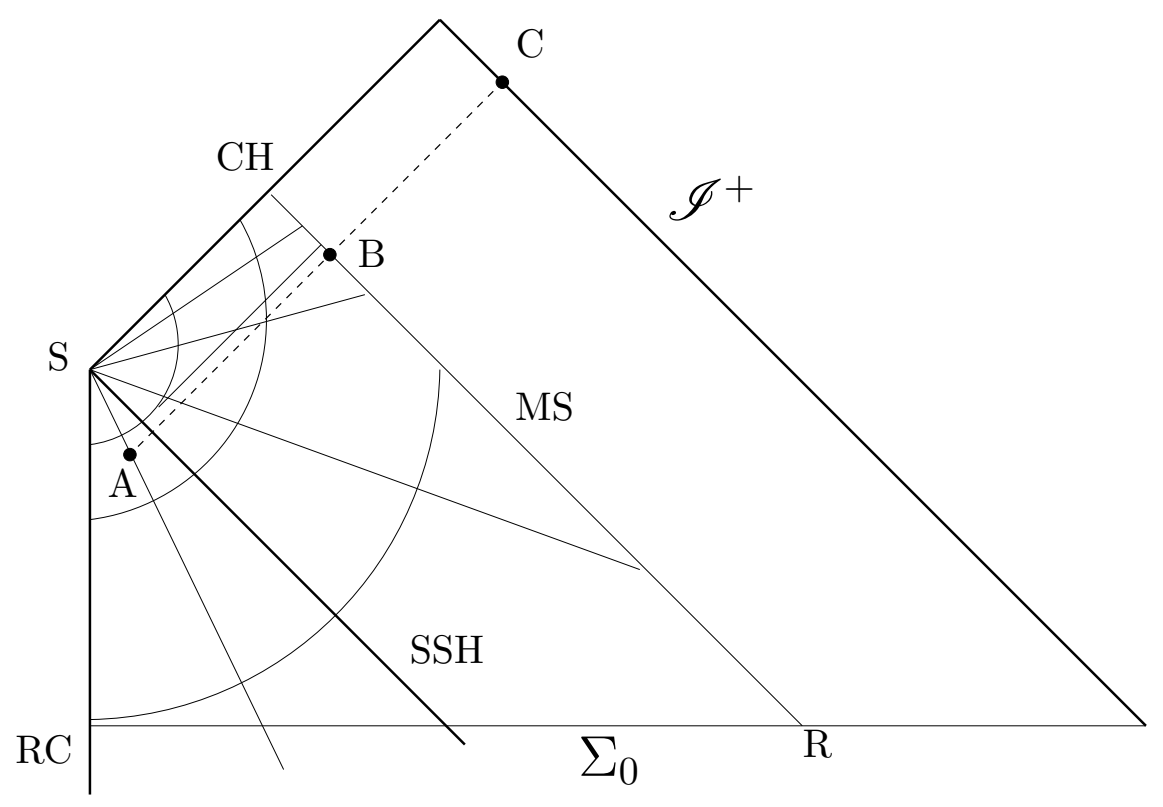

Figure 4: Conformal diagram of the critical solution matched to an asymptotically flat one. $\mathrm{RC}=$ regular centre, $\mathrm{S}=$ Singularity, $\mathrm{SSH}=$ self-similarity horizon. Curved lines are lines of constant coordinate $\tau$, while converging straight lines are lines of constant coordinate $x$. Let the initial data on the Cauchy surface CS be those for the exact critical solution out to the 2-sphere R, and let these data be smoothly extended to some data that are asymptotically flat, so that the future null infinity $\mathscr{I}^{+}$exists. To the past of the matching surface MS the solution coincides with the critical solution. The spacetime cannot be uniquely continued beyond the Cauchy horizon $\mathrm{CH}$. The redshift from point A to point B is finite by self-similarity, and the redshift from B to $\mathrm{C}$ is finite by asymptotic flatness. 


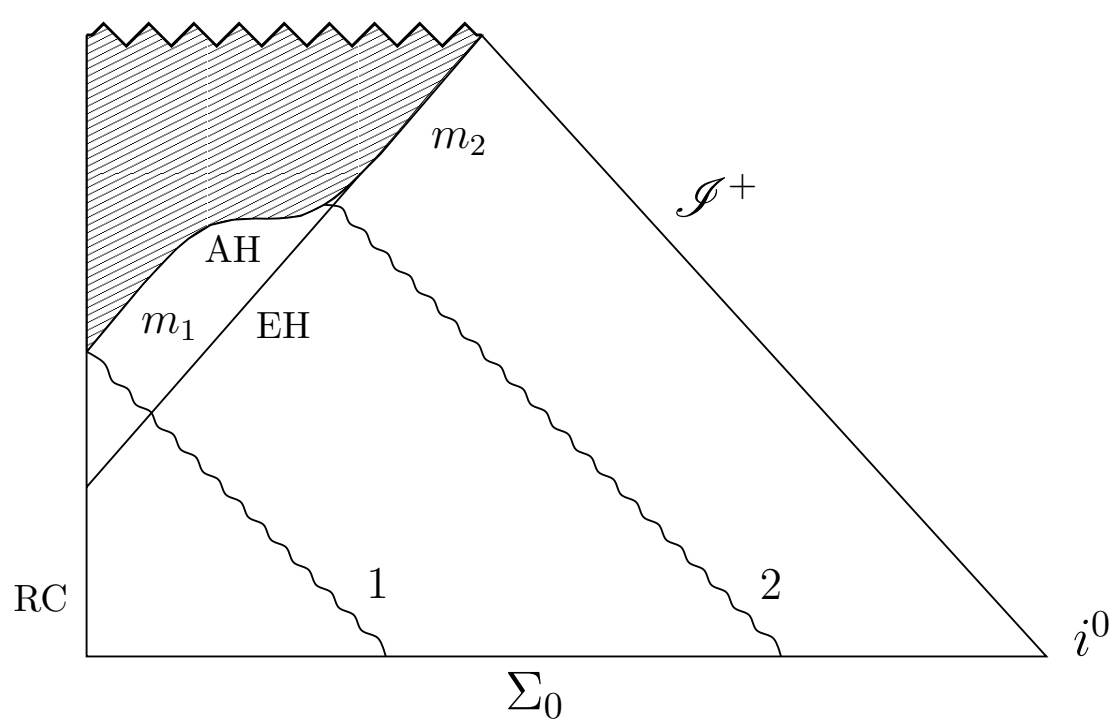

Figure 5: The final event horizon of a black hole is only known when the infall of matter has stopped. Radiation at 1 collapses to form a small black hole which settles down, but later more radiation at 2 falls in to give rise to a larger final mass. Fine-tuning of a parameter may result in $m_{1} \sim\left(p-p_{*}\right)^{\gamma}$, but the final mass $m_{2}$ would be approximately independent of $p$.

black hole charge

$$
Q \sim\left(p-p_{*}\right)^{\delta}
$$

was predicted. The predicted value $\delta \simeq 0.88$ of the critical exponent (in scalar electrodynamics) was subsequently verified in collapse simulations by Hod and Piran 121 and later again by Petryk [166]. (The mass scales with $\gamma \simeq 0.37$ as for the uncharged scalar field.) No other type of criticality can be found in the phase space of this system as dispersion and black holes are the only possible end states, though black holes with $|Q| \lesssim M$ can be formed [166].

General considerations similar to those in Sec. 2.6 led Gundlach and Martín-García to the general prediction that the two critical exponents are always related, for any matter model, by the inequality

$$
\delta \geq 2 \gamma
$$

(with the equality holding if the critical solution is charged), so that black hole charge can always be treated perturbatively at the black hole threshold. This has not yet been verified in any other matter model.

\subsection{Self-interaction potential}

An example of the richer phenomenology in the presence of a scale in the field equations is the spherical massive scalar field with a potential $m^{2} \phi^{2}$ [33] coupled to gravity: In one region of phase space, with characteristic scales smaller than $1 / m$, the black hole threshold is dominated by the Choptuik solution and type II critical phenomena occur. In another it is dominated by metastable oscillating boson stars (whose mass is of order $1 / m$ in geometric units) and type I critical phenomena occur. (For the real scalar field, the type I critical solution is an (unstable) 
oscillating boson star [175] while for the complex scalar field it can be a static (unstable) boson star [115.)

When the scalar field with a potential is coupled to electromagnetism, type II criticality is still controlled by a solution which asymptotically resembles the uncharged Choptuik spacetime, but type I criticality is now controlled by charged boson stars [166]. There are indications that subcritical type I evolutions lead to slow, large amplitude oscillations of stable boson stars 134, 135, 166 and not to dispersion to infinity, as had been conjectured in [115. Another interesting extension is the study of the dynamics of a real scalar field with a symmetric double-well potential, in which the system displays type I criticality between the two possible vacua [123.

\subsection{Nonspherical perturbations: stability and angular momentum}

Critical collapse is really relevant for cosmic censorship only if it is not restricted to spherical symmetry. Martín-García and Gundlach [146] have analysed all nonspherical perturbations of the scalar field critical solution by solving a linear eigenvalue problem with an ansatz of regularity at the centre and the SSH. They find that the only growing mode is the known spherical one, while all other spherical modes and all non-spherical modes decay. This strongly suggests that the critical solution is an attractor of codimension one not only in the space of spherically symmetric data but (modulo linearisation stability) of all data in a finite neighbourhood of spherical symmetry.

More recently, Choptuik and collaborators 52 have carried out axisymmetric time evolutions for the massless scalar field using adaptive mesh refinement. They find that in the limit of finetuning generic axisymmetric initial data the spherically symmetric critical solution is approached at first but then deviates from spherical symmetry and eventually develops two centres, each of which approaches the critical solution and bifurcates again in a universal way. This suggests that the critical solution has non-spherical growing perturbation modes, possibly a single $l=2$ even parity mode (in axisymmetry, only $m=0$ is allowed). There appears to be a conflict between the time evolution results [52] and the perturbative results [146, which needs to be resolved by more work (see Sec. [5.2).

Perturbing the scalar field around spherical symmetry, angular momentum comes in to second order in perturbation theory. All angular momentum perturbations were found to decay, and a critical exponent $\mu \simeq 0.76$ for the angular momentum was derived for the massless scalar field in 84. This prediction has not yet been tested in nonlinear collapse simulations.

\section{More spherical symmetry}

The pioneering work of Choptuik on the spherical massless scalar field has been followed by a plethora of further investigations. These could be organised under many different criteria. We have chosen the following rough categories:

- Systems in which the field equations, when reduced to spherical symmetry, form a single wave-like equation, typically with explicit $r$-dependence in its coefficients. This includes Yang-Mills fields, sigma models, vector and spinor fields, scalar fields in $2+1$ or in $4+1$ and more spacetime dimensions, and scalar fields in a semi-classical approximation to quantum gravity.

- Perfect fluid matter, either in an asymptotically flat or a cosmological context. The linearised Euler equations are in fact wave-like, but the full non-linear equations admit shock heating and are therefore not even time-reversal symmetric.

- Collisionless matter described by the Vlasov equation is a partial differential equation on particle phase space as well as spacetime. Therefore even in spherical symmetry, the matter 
equation is a partial differential equation in four dimensions (rather than two). Intuitively speaking, there are infinitely more matter degrees of freedom than in the scalar field or in non-spherical vacuum gravity.

- Spherically symmetric nonlinear wave equations on $(3+1)$ Minkowski spacetime, and other nonlinear partial differential equations which show a transition between singularity formation and dispersal.

Some of these examples were constructed because they may have intrinsic physical relevance (semiclassical gravity, primordial black holes), others as toy models for 3+1-dimensional gravity, and others mostly out of a purely mathematical interest. Table 1 gives an overview of these models.

\subsection{Matter obeying wave equations}

\subsubsection{2d nonlinear $\sigma$ model}

We have already discussed in subsection 3.6 the effects of adding a potential to the evolution of the scalar field. An alternative generalization is a modification of the kinetic term in the Lagrangian, with the general form

$$
\frac{1}{2} g^{\mu \nu} G_{I J}\left(\phi^{K}\right) \nabla_{\mu} \phi^{I} \nabla_{\nu} \phi^{J}
$$

for an N-dimensional vector field $\phi^{I}(x)$ with $I=1 . . N$, and where $G_{I J}\left(\phi^{K}\right)$ is a fixed nonlinear function acting as a metric on the so-called target space of the fields $\phi^{I}$. Such a system is called a nonlinear $\sigma$ model, harmonic map or wave map. The fields $\phi^{I}$ and metric $G_{I J}$ are dimensionless, and this allows the introduction of dimensionless parameters in the system, which cannot be asymptotically neglected using the arguments of subsection 2.6. (Compare with the potential $V\left(\phi^{I}\right)$, which has dimensions (length $)^{-2}$, and hence requires dimensionful parameters).

The case $N=1$ gives nothing new, and so Hirschmann and Eardly (HE from now on) studied the $N=2$ case with a target manifold with constant curvature 119, proportional to a real dimensionless constant $\kappa$. Using a single complex coordinate $\phi$ the action of the system can be written as

$$
\int d^{4} x \sqrt{g}\left[\frac{R}{2}-\frac{|\nabla \phi|^{2}}{\left(1-\kappa|\phi|^{2}\right)^{2}}\right] .
$$

For $\kappa \geq 0$ this system is equivalent to the problem of a real massless scalar field coupled to BransDicke (BD) gravity (with the BD coupling constant given by $8 \omega_{B D}=-12+\kappa^{-1}$ ). Liebling and Choptuik [143] have shown that there is a smooth transition in the BD system from DSS criticality for low $\kappa$ (the flat target space case $\kappa=0$ is equivalent to a self-gravitating complex massless scalar field, whose critical solution is the original DSS spacetime found by Choptuik) to CSS criticality for larger $\kappa$ (the case $\kappa=1$ is equivalent to the axion-dilaton system, and has been shown to display CSS criticality in [106]).

Generalizing their previous results for $\kappa=0$ [118, 117, HE constructed for each $\kappa$ a CSS solution based on the ansatz $\phi(\tau, x)=e^{i \omega \tau} \phi(x)$ for the critical scalar field. Studying its perturbations HE concluded that this solution is critical for $\kappa>0.0754$, but has three unstable modes for $\kappa<0.0754$ and even more for $\kappa<-0.28$. Below 0.0754 a DSS solution takes over, as shown in the simulations of Liebling and Choptuik [143, and HE conjectured that the transition is a Hopf bifurcation, such that the DSS cycle smoothly shrinks with growing $\kappa$, collapsing onto the CSS solution at the transition and then disappearing with a finite value of the echoing period $\Delta$.

The close relation between the CSS and DSS critical solutions is also manifest in the construction of their global structures. In particular, the results of [117] and 67] for the CSS $\kappa=0$ and $\kappa=1$ solutions respectively show that the Cauchy horizon of the singularity is almost but not quite flat, exactly as was the case with the Choptuik DSS spacetime (see subsection 3.3). 


\subsubsection{Spherical Einstein- $S U(2)$ sigma model}

This is an $N=3$ sigma model, and it also displays a transition between CSS and DSS criticality, but this is a totally different type of transition, in particular showing a divergence in the echoing period $\Delta$ [126].

In a reduction to spherical symmetry, the effective action is

$$
\int d^{4} x \sqrt{g}\left[\frac{R}{2}-\eta\left(|\nabla \phi|^{2}+\frac{2 \sin ^{2} \phi}{r^{2}}\right)\right],
$$

where $r$ is the area radius, and the coupling constant $\eta$ is dimensionless. It has been shown (numerically [25] and then analytically [26]) that for $0 \leq \eta<1 / 2$, there is an infinite sequence of CSS solutions $\phi_{n}$ labelled by a nodal number $n$, and having $n$ growing modes. (The case $\eta=0$, in which the sigma field decouples from gravity, will be revisited below.) The $n$-th solution is always regular in the past light cone of the singularity, but is regular up to the future light cone only for $\eta<\eta_{n}$ where $\eta_{0} \simeq 0.0688, \eta_{1} \simeq 0.1518$ and $\eta_{n}<\eta_{n+1}<1 / 2$. For larger couplings an apparent horizon develops and the solution cannot be smoothly continued. These results suggest that $\phi_{0}$ is a stable naked singularity for $\eta<\eta_{0}$, and $\phi_{1}$ acts as a critical solution between naked singularity formation and dispersal for $\eta<\eta_{0}$ and between black hole formation and dispersal for $\eta_{0}<\eta<\eta_{1}$. The numerical experiments agree with this scenario in the range $0 \leq \eta<0.14$. Other CSS solutions of this system are investigated in [23], and the possibility of chaos in [182].

Aichelburg and collaborators [126, 137, have shown that for $\eta \gtrsim 0.2$ there is clear DSS type II criticality at the black hole threshold. The period $\Delta$ depends on $\eta$, monotonically decreasing towards an asymptotic value for $\eta \rightarrow \infty$. Interesting new behavior occurs in the intermediate range $0.14<\eta<0.2$ that lies between clear CSS and clear DSS. With decreasing $\eta$ the overall DSS includes episodes of approximate CSS [183, of increasing length (measured in the log-scale time $\tau$ ). As $\eta \rightarrow \eta_{c} \simeq 0.170$ from above the duration of the CSS epochs, and hence the overall DSS period $\Delta$ diverges. For $0.14<\eta<0.17$ time evolutions of initial data near the black hole threshold no longer show overal DSS, but they still show CSS episodes. Black hole mass scaling is unclear in this regime.

It has been conjectured that this transition from CSS to DSS can be interpreted, in the language of the theory of dynamical systems, as the infinite-dimensional analogue of a three-dimensional Shil'nikov bifurcation 138. High-precision numerics in 3 further supports this picture: for $\eta>\eta_{c}$ a codimension-1 CSS solution coexists in phase space with a codimension-1 DSS attractor such that the (one-dimensional) unstable manifold of the DSS solution lies on the stable manifold of the CSS solution. For $\eta$ close to $\eta_{c}$ the two solutions are close and the orbits around the DSS solution become slower because they spend more time in the neighbourhood of the CSS attractor. A linear stability analysis predicts a law $\Delta \simeq-\frac{2}{\lambda} \log \left(\eta-\eta_{c}\right)+b$ for some constant $b$, where $\lambda$ is the Lyapunov exponent of the CSS solution. For $\eta=\eta_{c}$ both solutions touch and the DSS cycle dissapears.

\subsubsection{Einstein-Yang-Mills}

Choptuik, Chmaj and Bizoń 50 , have found both type I and type II critical collapse in the spherical Einstein-Yang-Mills system with SU(2) gauge potential, restricting to the purely magnetic case, in which the matter is described by a single real scalar field. The situation is very similar to that of the massive scalar field, and now the critical solutions are the well-known static $n=1$ Bartnik-Mckinnon solution [8] for type I and a DSS solution (later constructed in 93]) for type II. In both cases the black holes produced in the supercritical regime are Schwarzschild black holes with zero Yang-Mills field strength, but the final states (and the dynamics leading to them) can

be distinguished by the value of the Yang-Mills final gauge potential at infinity, which can take two values, corresponding to two distinct vacuum states. 
Choptuik, Hirschmann and Marsa [54 have investigated the boundary in phase space between formation of those two types of black holes, using a code that can follow the time evolutions for long after the black hole has formed. This is a new "type III" phase transition whose critical solution is an unstable static black hole with Yang-Mills hair [12, 188, which collapses to a hairless Schwarzschild black hole with either vacuum state of the Yang-Mills field, depending on the sign of its one growing perturbation mode. This "colored" black hole is actually a member of a 1-parameter family parameterized by its apparent horizon radius and outside the horizon it approaches the corresponding BM solution. When the horizon radius approaches zero the three critical solutions meet at a "triple point". What happens there deserves further investigation.

Millward and Hirschmann 150 have further coupled a Higgs field to the Einstein-Yang-Mills system. New possible end states appear: regular static solutions, and stable hairy black holes (different from the colored black holes referred to above). Again there are type I or type II critical phenomena depending on the initial conditions.

It is known that the spherical critical solutions within the magnetic ansatz become more unstable when other components of the gauge field are taken into account, and so they will not be critical in the general case.

\subsubsection{Vacuum $4+1$}

Bizoń, Chmaj and Schmidt [18 have found a way of constructing asymptotically flat vacuum spacetimes in $4+1$ dimensions which are spherically symmetric while containing gravitational waves (Birkhoff's theorem does not hold in more than $3+1$ dimensions). Recall that in (3+1 dimensional) Bianchi IX cosmology the manifold is $M^{1} \times S^{3}$ where the $S^{3}$ is equipped with an $S U(2)$ invariant (homogeneous but anisotropic) metric

$$
d s^{2}=L_{1}^{2} \sigma_{1}^{2}+L_{2}^{2} \sigma_{3}^{2}+L_{3}^{2} \sigma_{3}^{2},
$$

where the $\sigma_{i}$ are the $S U(2)$ left-invariant 1-forms, and the $L_{i}$ are functions of time only. Similarly, the spacetimes of Bizon, Chmaj and Schmidt are of the product form $M^{2} \times S^{3}$ where the $L_{i}$ now depend only on $r$ and $t$. This gives rise to nontrivial dynamics, including a threshold between dispersion and black hole formation. With the additional $U(1)$ symmetry $L_{1}=L_{2}$ there is only one dynamical degree of freedom. At the black hole threshold, type II critical phenomena are seen with $\Delta \simeq 0.49$ and $\gamma \simeq 0.3289$. In evolutions with the general ansatz where all $L_{i}$ are different, the $U(1)$ symmetry is recovered dynamically [19. Furthermore, the fact that each $U(1)$-symmetric solution exists in 3 copies gives rise to additional critical surfaces, and this lead to the prediction of 2-mode-unstable solutions, which was verified numerically, and the conjecture that there is a countable family of DSS solutions with $n$ unstable modes [19. A similar ansatz can be made in other odd spacetime dimensions, and in $8+1$ dimensions type II critical behaviour is again observed [17.

\subsubsection{Scalar field collapse in $2+1$}

Spacetime in $2+1$ dimensions is flat everywhere where there is no matter, so that gravity is not acting at a distance in the usual way. There are no gravitational waves, and black holes can only be formed in the presence of a negative cosmological constant. (See [37] for a review.)

Scalar field collapse in circular symmetry was investigated numerically by Pretorius and Choptuik [168, and Husain and Olivier [128. In a regime where the cosmological constant is small compared to spacetime curvature they find type II critical phenomena with a universal CSS critical solution, and $\gamma=1.20 \pm 0.05$ [168]. The value $\gamma \simeq 0.81$ [128] appears to be less accurate.

Looking for the critical solution in closed form, Garfinkle [79] found a countable family of exact spherically symmetric CSS solutions for a massless scalar field with $\Lambda=0$, but his results remain 
inconclusive. The $q=4$ solution appears to match the numerical evolutions inside the past light cone, but its past light cone is also an apparent horizon. The $q=4$ solution has three growing modes although the top one would give $\gamma=8 / 7 \simeq 1.14$ if only the other two could be ruled out 83. An attempt at this 120 seems unmotivated. At the same time, it is possible to embed the $\Lambda=0$ solutions into a family of $\Lambda<0$ ones [62, 63, 41, which can be constructed along the lines of Sec. 2.6. so that Garfinkle's solution could be the leading term in an expansion in $e^{-\tau}(-\Lambda)^{-1 / 2}$.

\subsubsection{Scalar field collapse in higher dimensions}

Critical collapse of a massless scalar field in spherical symmetry in $5+1$ spacetime dimensions was investigated in 80 . Results are similar to $3+1$ dimensions, with a DSS critical solution and mass scaling with $\gamma \simeq 0.424$. Birukou et al [11, 127] have developed a code for arbitrary spacetime dimension. They confirm known results in $3+1(\gamma \simeq 0.36)$ and $5+1(\gamma \simeq 0.44)$ dimensions, and investigate $4+1$ dimensions. Without a cosmological constant they find mass scaling with $\gamma \simeq 0.41$ for one family of initial data and $\gamma \simeq 0.52$ for another. They see wiggles in the $\ln M$ versus $\ln (p-p *)$ plot that indicate a DSS critical solution, but have not investigated the critical solution directly. With a negative cosmological constant and the second family, they find $\gamma=0.49$. 27. has made a more precise determination: $\gamma=0.4131 \pm 0.0001$. This was motivated by an attempt to explain this exponent using an holographic duality between the strong coupling regime of $4+1$ gravity and the weak coupling regime of 3+1 QCD [5], which had predicted $\gamma=0.409552$.

Kol 133 relates a solution that is related to the Choptuik solution to a variant of the critical solution in the black-string black hole transition, and claims to obtain analytic estimates for $\gamma$ and $\Delta$. This has motivated a numerical determination of $\gamma$ and $\Delta$ for the spherical massless scalar field in noninteger dimension up to 14 [178, 28].

\subsubsection{Other systems obeying wave equations}

Choptuik, Hirschmann and Liebling [51 have presented perturbative indications that the static solutions found by van Putten [185] in the vacuum Brans-Dicke system are critical solutions. They have also performed full numerical simulations, but only starting from small deviations with respect to those solutions.

Ventrella and Choptuik [186 have performed numerical simulations of collapse of a massless Dirac field in a special state: an incoherent sum of two independent left-handed zero-spin fields having opposite orbital angular momentum. This is prepared so that the total distribution of energy-momentum is spherically symmetric. The freedom in the system is then contained in a single complex scalar field obeying a modified linear wave equation in spherical symmetry. There are clear signs of CSS criticality in the metric variables, and the critical complex field exhibits a phase of the form $e^{i \omega \tau}$ for a definite $\omega$ (the Hirschmann and Eardly ansatz for the complex scalar field critical solutions), which can be considered as a trivial form of DSS.

Garfinkle, Mann and Vuille [86] have found coexistence of types I and II criticality in the spherical collapse of a massive vector field (the Proca system), the scenario being almost identical to that of a massive scalar field. In the self-similar phase the collapse amplifies the longitudinal

mode of the Proca field with respect to its transverse modes, which become negligible, and the critical solution is simply the gradient of the Choptuik DSS spacetime.

Sarbach and Lehner [174] find type I critical behaviour in $q+3$-dimensional spacetimes with $U(1) \times S O(q+1)$ symmetry in Einstein-Maxwell theory at the threshold between dispersion and formation of a black string. 
Table 1: Critical collapse in spherical symmetry

\begin{tabular}{|c|c|c|c|c|}
\hline Matter & Type & $\begin{array}{l}\text { Collapse } \\
\text { simulations }\end{array}$ & $\begin{array}{l}\text { Critical } \\
\text { solution }\end{array}$ & $\begin{array}{l}\text { Perturbations } \\
\text { of crit. soln. }\end{array}$ \\
\hline $\begin{array}{l}\text { Perfect fluid } p=k \rho \\
\text { Vlasov }\end{array}$ & $\begin{array}{l}\mathrm{II} \\
\text { I? }\end{array}$ & \begin{tabular}{l|l|}
69 & 152 \\
172 & 159 \\
172
\end{tabular} & CSS $\frac{699,145,152]}{147]}$ & 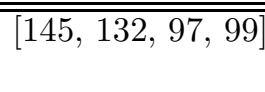 \\
\hline $\begin{array}{l}\text { Real scalar field: } \\
- \text { massless, min. coupled } \\
- \text { massive } \\
- \text { conformally coupled } \\
-4+1 \\
-5+1\end{array}$ & $\begin{array}{l}\text { II } \\
\text { I } \\
\text { II } \\
\text { II } \\
\text { II } \\
\text { II }\end{array}$ & 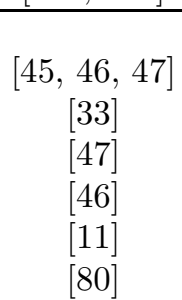 & $\begin{array}{c}\text { DSS }[91] \\
\text { oscillating }[175 \\
\text { DSS }[108,102] \\
\text { DSS }\end{array}$ & $\begin{array}{l}\text { 94, } 146] \\
108,102]\end{array}$ \\
\hline $\begin{array}{l}\text { Massive complex scalar field } \\
\text { Massless scalar electrodynamics }\end{array}$ & $\begin{array}{c}\text { I, II } \\
\text { II }\end{array}$ & 115 & $\begin{array}{c}175] \\
\text { DSS } 102\end{array}$ & $\begin{array}{l}115 \\
102\end{array}$ \\
\hline $\begin{array}{l}\text { Massive vector field } \\
\text { Massless Dirac } \\
\text { Vacuum Brans-Dicke }\end{array}$ & $\begin{array}{c}\text { II } \\
\text { II } \\
\text { I }\end{array}$ & $\frac{866}{186}$ & $\begin{array}{l}\text { DSS }[86] \\
\text { CSS }[186] \\
\text { static } 185\end{array}$ & 56 \\
\hline $\begin{array}{l}\text { 2-d sigma model } \\
\text { - complex scalar }(\kappa=0) \\
\text { - axion-dilaton }(\kappa=1) \\
\text { - scalar-Brans-Dicke }(\kappa>0) \\
\text { - general } \kappa \text { including } \kappa<0\end{array}$ & $\begin{array}{l}\text { II } \\
\text { II } \\
\text { II } \\
\text { II }\end{array}$ & $\begin{array}{c}44 \\
106 \\
143,140\end{array}$ & $\begin{array}{c}\text { DSS } 94] \\
\text { CSS }[67,106 \\
\text { CSS, DSS } \\
\text { CSS, DSS } 119\end{array}$ & $\begin{array}{l}94 \\
106 \\
119 \\
19\end{array}$ \\
\hline $\begin{array}{l}S U(2) \text { Yang-Mills } \\
S U(2) \text { Yang-Mills-Higgs } \\
S U(2) \text { Skyrme model } \\
S O(3) \text { Mexican hat }\end{array}$ & $\begin{array}{c}\text { I } \\
\text { II } \\
\text { "III" } \\
\text { (idem) } \\
\text { I } \\
\text { II } \\
\text { II }\end{array}$ & \begin{tabular}{|l|}
50 \\
50 \\
54 \\
150 \\
15 \\
20 \\
141 \\
\end{tabular} & $\begin{array}{c}\text { static [8] } \\
\text { DSS [93] } \\
\text { colored BH [12, 188] } \\
\text { (idem) } \\
\text { static [15] } \\
\text { DSS } 20] \\
\text { DSS }\end{array}$ & $\begin{array}{c}{[136]} \\
{[180,187,16]} \\
15\end{array}$ \\
\hline
\end{tabular}




\subsection{Perfect fluid matter}

\subsubsection{Spherical symmetry}

Evans and Coleman 69. performed the first simulations of critical collapse with a perfect fluid with equation of state $p=k \rho$ (where $\rho$ is the energy density and $p$ the pressure) for $k=1 / 3$ (radiation), and found a CSS critical solution with a mass-scaling critical exponent $\gamma \approx 0.36$. Koike, Hara and Adachi 131 constructed that critical solution and its linear perturbations from a CSS ansatz as an eigenvalue problem, computing the critical exponent to high precision. Independently, Maison [145] constructed the regular CSS solutions and their linear perturbations for a large number of values of $k$, showing for the first time that the critical exponents were model-dependent. As Ori and Piran before 161, 162, he claimed that there are no regular CSS solutions for $k>0.89$, but Neilsen and Choptuik [152, 153 have found CSS critical solutions for all values of $k$ right up to 1, both in collapse simulations and by making a CSS ansatz. The difficulty comes from a change in character of the sonic point, which becomes a nodal point for $k>0.89$, rather than a focal point, making the ODE problem associated with the CSS ansatz much more difficult to solve. Harada [110] has also found that the critical solution becomes unstable to a "kink" (discontinuous at the sonic point of the background solution) mode for $k>0.89$, but because it is not smooth it does not seem to have any influence on the numerical simulations of collapse. On the other hand, the limit $k \rightarrow 1$ leading to the stiff equation of state $p=\rho$ is singular in that during evolution the fluid 4 -velocity can become spacelike and the density $\rho$ negative. The stiff fluid equations of motion are in fact equivalent to the massless-scalar field, but the critical solutions can differ, dependending on how one deals with the issue of negative density [30. Summarizing, it is possible to construct the Evans-Coleman CSS critical (codimension 1) solution for all values $0<k<1$. This solution can be identified in the general classification of CSS perfect-fluid solutions as the unique spacetime that is analytic at the center and at the sound cone, is ingoing near the center, and outgoing everywhere else [38, 39, 40. There is even a Newtonian counterpart of the critical solution: the Hunter(a) solution [112.

$p=k \rho$ is the only equation of state compatible with exact CSS (homothetic) solutions for perfect fluid collapse [36] and therefore we might think that other equations of state would not display critical phenomena, at least of type II. Neilsen and Choptuik [152] have given evidence that for the ideal gas equation of state (from now on EOS) $p=k \rho_{0} \epsilon$ (where $\rho_{0}$ is the rest mass density and $\epsilon$ is the internal energy per rest mass unit) the black hole threshold also contains a CSS attractor, and that it coincides with the CSS exact critical solution of the ultrarelativistic case with the same $k$. This is interpreted a posteriori as a sign that the critical CSS solution is highly ultrarelativistic $\rho=(1+\epsilon) \rho_{0} \simeq \epsilon \rho_{0} \gg \rho_{0}$, and hence rest mass is irrelevant. Novak [158, has also shown in the case $k=1$, or even with a more general tabulated EOS, that type II critical phenomena can be found by velocity-induced perturbations of static TOV solutions. A thorough and much more precise analysis by Noble and Choptuik [156, 157. of the possible collapse scenarios of the stiff $k=1$ ideal gas has confirmed this surprising result, and again the critical solution (and hence the critical exponent) is that of the ultrarelativistic limit problem. Parametrizing, as usual, the TOV solutions by the central density $\rho_{c}$, they find that for low-density initial stars it is not possible to form a black hole by velocity-induced collapse; for intermediate initial values of $\rho_{c}$, it is possible to induce type II criticality for large enough velocity perturbations; for large initial central densities they always get type I criticality, as we might have anticipated.

Noble and Choptuik [156] have also investigated the evolution of a perfect fluid interacting with a massless scalar field indirectly through gravity. By tuning of the amplitude of the pulse it is possible to drive a fluid star to collapse. For massive stars type I criticality is found, in which the critical solution oscillates around a member of the unstable TOV branch. For less massive stars a large scalar amplitude is required to induce collapse, and the black hole threshold is always dominated by the scalar field DSS critical solution, with the fluid evolving passively. 


\subsubsection{Nonspherical perturbations}

Non-spherically symmetric perturbations around the spherical critical solution for the perfect fluid can be used to study angular momentum perturbatively. All nonspherical perturbations of the perfect fluid critical solution decay for equations of state $p=k \rho$ with $k$ in the range $1 / 9<k<$ 0.49 [97, and so the spherically symmetric critical solution is stable under small deviations from spherical symmetry. Infinitesimal angular momentum is carried by the axial parity perturbations with angular dependence $l=1$. From these two facts one can derive the angular momentum scaling law at the black hole threshold [95, 99]

$$
L \sim\left(p-p_{*}\right)^{\mu},
$$

which should be valid in the range $1 / 9<k<0.49$. The angular momentum exponent $\mu(k)$ is related to the mass exponent $\gamma(k)$ by

$$
\mu(k)=\left(2+\lambda_{1}(k)\right) \gamma(k) .
$$

where

$$
\lambda_{1}(k)=\frac{9 k-1}{3 k+3}
$$

is the growth or decay rate of the dominant $l=1$ axial perturbation mode. In particular for the value $k=1 / 3$, where $\gamma \simeq 0.3558, \mu=(5 / 2) \gamma \simeq 0.8895$.

Ori and Piran [162] have pointed out that there exists a CSS perfect fluid solution for $0<k<$ 0.036 generalizing the Larson-Penston solution of Newtonian fluid collapse, and which has a naked singularity for $0<k<0.0105$. Harada and Maeda [109, 112] have shown that this solution has no growing perturbative modes in spherical symmetry and hence a naked singularity becomes a global attractor of the evolution for the latter range of $k$. This is also true in the limit $k=0$, which can be considered as the Newtonian limit [113] 111]. Their result has been confirmed with very high precision numerics by Snajdr [177. This seems to violate cosmic censorship, as generic spherical initial data would create a naked singularity. However, the exact result (40) holds for any regular CSS spherical perfect fluid solution, and so all such solutions with $k<1 / 9$ have at least one unstable nonspherical perturbation. Therefore the naked singularity is unstable to infinitesimal perturbations with angular momentum when one lifts the restriction to spherical symmetry.

In the early universe, quantum fluctuations of the metric and matter can be important, for example providing the seeds of galaxy formation. Large enough fluctuations will collapse to form primordial black holes. As large quantum fluctuations are exponentially more unlikely than small ones, $P(\delta) \sim e^{-\delta^{2}}$, where $\delta$ is the density contrast of the fluctuation, one would expect the spectrum of primordial black holes to be sharply peaked at the minimal $\delta$ that leads to black hole formation, giving rise to critical phenomena [154. See also [90, 194].

An approximation to primordial black hole formation is a spherically symmetric distribution of a radiation gas $(p=\rho / 3)$ with cosmological rather than asymptotically flat boundary conditions. In 154, 155 type II critical phenomena were found, which would imply that the mass of primordial black holes formed are much smaller than the naively expected value of the mass contained within the Hubble horizon at the time of collapse. The boundary conditions and initial data were refined in [114, 151, and a minimum black hole mass of $\sim 10^{-4}$ of the horizon mass was found, due to matter accreting onto the black hole after strong shock formation.

\subsection{Collisionless matter}

A cloud of collisionless particles can be described by the Vlasov equation, i.e., the Boltzmann equation without collision term. This matter model differs from field theories by having a much 
larger number of matter degrees of freedom: The matter content is described by a statistical distribution $f\left(x^{\mu}, p_{\nu}\right)$ on the point particle phase space, instead of a finite number of fields $\phi\left(x^{\mu}\right)$. When restricted to spherical symmetry, individual particles move tangentially as well as radially, and so individually have angular momentum, but the stress-energy tensor averages out to a spherically symmetric one, with zero total angular momentum. The distribution $f$ is then a function $f\left(r, t, p^{r}, L^{2}\right)$ of radius, time, radial momentum and (conserved) angular momentum.

Several numerical simulations of critical collapse of collisionless matter in spherical symmetry have been published to date, and remarkably no type II scaling phenomena has been discovered. Indications of type I scaling have been found, but these do not quite fit the standard picture of critical collapse. Rein et al. [172] find that black hole formation turns on with a mass gap that is a large part of the ADM mass of the initial data, and this gap depends on the initial matter condition. No critical behavior of either type I or type II was observed. Olabarrieta and Choptuik [159] find evidence of a metastable static solution at the black hole threshold, with type I scaling of its life time as in Eq. (13). However, the critical exponent depends weakly on the family of initial data, ranging from 5.0 to 5.9, with a quoted uncertainty of 0.2. Furthermore, the matter distribution does not appear to be universal, while the metric seems to be universal up to an overall rescaling, so that there appears to be no universal critical solution. More precise computations by Stevenson and Choptuik [179, using finite volume HRSC methods, have confirmed the existence of static intermediate solutions and non-universal scaling with exponents ranging now from 5.27 to 11.65 .

Martín-García and Gundlach [147] have constructed a family of CSS spherically symmetric solutions for massless particles that is generic by function counting. There are infinitely many solutions with different matter configurations but the same stress-energy tensor and spacetime metric, due to the existence of an exact symmetry: two massless particles with energy-momentum $p^{\mu}$ in the solution can be replaced by one particle with $2 p^{\mu}$. A similar result holds for the perturbations. As the growth exponent $\lambda$ of a perturbation mode can be determined from the metric alone, this means that there are infinitely many perturbation modes with the same $\lambda$. If there is one growing perturbative mode, there are infinitely many. Therefore a candidate critical solution (either static or CSS) cannot be isolated or have only one growing mode. This argument rules out the existence of both type I and type II critical phenomena (in their standard form, i.e., including universality) for massless particles in the complete system, but some partial form of criticality could still be found by restricting to sections of phase space in which that symmetry is broken, for example by prescribing a fixed form for the dependence of the distribution function $f$ on angular momentum $L$, as those numerical simulations have done.

A recent investigation of Andréasson and Rein [ 6 with massive particles has confirmed again the existence of a mass gap and the existence of metastable static solutions at the black hole threshold, though there is no estimation of the scaling of their life-times. More interestingly, they show that the subcritical regime can lead to either dispersion or an oscillating steady state depending on the binding energy of the system. They also conclude, based on perturbative arguments, that there cannot be an isolated universal critical solution.

More numerical work is still required, but current evidence suggests that there are no type II critical phenomena, and that there is a continuum of critical solutions in type I critical phenomena and hence only limited universality.

\subsection{Criticality in singularity formation without gravitational collapse}

It is well known that the Yang-Mills field does not form singularities from smooth initial conditions in 3+1 dimensions [68, but Bizon and Tabor [24] have shown singularity formation in $4+1$ (the critical dimension for this system from the point of view of energy scaling arguments) and $5+1$ (the first supercritical dimension). In $5+1$ there is countable family $W_{n}$ of CSS solutions with $n$ 
unstable modes, such that $W_{1}$ acts as a critical solution separating singularity $\left(W_{0}\right)$ formation from dispersal to infinity. In $4+1$ there are no self-similar solutions and the formation of singularities seems to proceed through adiabatic shrinking of a static solution.

Completely parallel results can be found for wave maps, for which the critical dimension is $2+1$. For the wave map from 3+1 Minkowski to the 3-sphere Bizon [14 has shown that there is a countable family of regular (before the Cauchy horizon) CSS solutions labeled by a nodal number $n \geq 0$, such that each solution has $n$ unstable modes. Simulations of collapse in spherical symmetry 21, 144 and in 3D 142 show that $n=0$ is a global attractor and the $n=1$ solution is the critical solution. See also [65, 66] for computations of the largest perturbation-eigenvalues of $W_{0}$ and $W_{1}$. Again, for the wave map from 2+1 Minkowski to the 2-sphere generic singularity formation proceeds through adiabatic shrinking of a static solution 22 .

These results have led to the suggestion in [24] that criticality (in the sense of the existence of a codimension-1 solution separating evolution towards qualitatively different end states) could be a generic and robust feature of evolutionary PDE systems in supercritical dimensions, and not an effect particular of gravity.

Garfinkle and Isenberg [85] examine the threshold between the round endstate and pinching off in Ricci flow for a familiy of spherically symmetric geometries on $S^{3}$. They have found intermediate approach to a special "javelin" geometry, but have not investigated whether this is universal.

A scaling of the shape of the event horizon at the moment of merger in binary black hole mergers is noted in [4], but is really a kinematic effect.

\subsection{Analytic studies and toy models}

\subsubsection{Exact solutions of Einstein-Klein-Gordon}

A number of authors have explored the possibility of finding critical phenomena with CSS (rather than DSS) massless scalar critical solutions. The Roberts 1-parameter family of $3+1$ solutions [173. has been analyzed along this line in 163, 31, 191, 130. This family contains black holes whose masses (with a suitable matching to an asymptotically flat solution) scale as $(p-1)^{1 / 2}$ for $p \gtrsim 1$, but such a special family of solutions has no direct relevance for collapse from generic data. Its generalization to other dimensions has been considered in [72. A fully analytic construction of all (spherical and nonspherical) linear perturbations of the Roberts solution by Frolov [70, 71, has shown that there is a continuum of unstable spherical modes filling a sector of the complex plane with $\operatorname{Re} \lambda \geq 1$, so that it cannot be a critical solution. Interestingly, all nonspherical perturbations decay.

Frolov 73 has also suggested that the critical $(p=1)$ Roberts solution, which has an outgoing null singularity, plus its most rapidly growing (spherical) perturbation mode would evolve into the Choptuik solution, which would inherit the oscillation in $\tau$ with a period 4.44 of that mode.

A similar transition within a single 1-parameter family of solutions has been pointed out in [160] for the Wyman solution [192].

Hayward [116, 64 and Clement and Fabbri 62, 63] have also proposed critical solutions with a null singularity, and have attempted to construct black hole solutions from their linear perturbations. This is probably irrelevant to critical collapse, as the critical spacetime does not have an outgoing null singularity. Rather, the singularity is naked but first appears in a point. The future light cone of that point is not a null singularity but a Cauchy horizon with finite curvature.

Other authors have attempted analytic approximations to the Choptuik solution. Pullin [170] has suggested describing critical collapse approximately as a perturbation of the Schwarzschild spacetime. Price and Pullin [169] have approximated the Choptuik solution by two flat space solutions of the scalar wave equation that are matched at a "transition edge" at constant selfsimilarity coordinate $x$. The nonlinearity of the gravitational field comes in through the matching procedure, and its details are claimed to provide an estimate of the echoing period $\Delta$. 


\subsubsection{Toy models}

Birmingham and Sen [10] considered the formation of a black hole from the collision of two point particles of equal mass in $2+1$ gravity. Peleg and Steif [165] have investigated the collapse of a dust ring. In both cases the mass of the black holes is a known function of the parameters of the initial condition, giving a "critical exponent" $1 / 2$, but no underlying self-similar solution is involved.

Frolov [75] and Frolov, Larsen and Christensen [76] consider a stationary 2+1-dimensional Nambu-Goto membrane held fixed at infinity in a stationary 3+1-dimensional black hole background spacetime. The induced $2+1$ metric on the membrane can have wormhole, black hole, or Minkowski topology. The critical solution between Minkowski and black hole topology has $2+1$ CSS. The mass of the apparent horizon of induced black hole metrics scales with $\gamma=2 / 3$, superimposed with a wiggle of period $3 \pi / \sqrt{7}$ in $\ln p$. The mass scaling is universal with respect to different background black hole metrics, as they can be approximated by Rindler space in the mass scaling limit.

Horowitz and Hubeny [125] and Birmingham [9] have attempted to calculate the critical exponent in toy models from the adS-CFT correspondence.

Burko [35] considers the transition between existence and non-existence of a null branch of the singularity inside a spherically symmetric charged black hole with massless scalar field matter thrown in.

Wang [190] has constructed homothetic cylindrically symmetric solutions of $3+1$ Einstein-KleinGordon and studied their cylindrically symmetric perturbations. It is not clear how these are related to a critical surface in phase space.

\subsection{Quantum effects}

Type II critical phenomena provide a relatively natural way of producing arbitrarily high curvatures, where quantum gravity effects should become important, from generic initial data. Approaching the Planck scale from above, one would expect to be able to write down a critical solution that is the classical critical solution asymptotically at large scales, as an expansion in inverse powers of the Planck length, see Sec2.6

Black hole evolution in semiclassical gravity has been investigated in $1+1$ dimensional models which serve as toy models for spherical symmetry (see 88 for a review). The black hole threshold in such models has been investigated in [43, 77, 181, [130, 195], 164, 29. In some of these models, the critical exponent is $1 / 2$ for kinematical reasons.

In [34] a 3+1-dimensional but perturbative approach is taken. The quantum effects then give an additional unstable mode with $\lambda=2$. If this is larger than the positive Lyapunov exponent $\lambda_{0}$, it will become the dominant perturbation for sufficiently good fine-tuning, and therefore sufficiently good fine-tuning will reveal a mass gap.

\section{$5 \quad$ Beyond spherical symmetry}

Numerical studies of critical collapse should go beyond spherical symmetry (and in the first instance to axisymmetry) for three reasons:

- Weak gravitational waves in vacuum general relativity can focus and collapse. The black hole threshold in this process shows what in critical phenomena in gravitational collapse is intrinsic to gravity rather than the matter model.

- Black holes are characterised by charge and angular momentum as well as mass. Angular momentum is the more interesting of the two because it is again independent of matter, but cannot be studied in spherical symmetry. 
- Angular momentum resists collapse, but angular momentum in the initial data is needed to make a black hole with angular momentum. Therefore it is an interesting question to ask what happens to the dimensionless ratio $J / M^{2}$ at the black hole threshold.

In the following we review what has been done so far.

\subsection{Perturbative approach to angular momentum}

We have already mentioned that when angular momentum is small, a critical exponent for $|J|$ can be derived in perturbation theory. This has been done for the perfect fluid (see Sec. 4.2) in first-order perturbation theory and for the massless scalar field (see Sec. 3.7), where second-order perturbation theory in the scalar field is necessary to obtain an angular momentum perturbation in the stress-energy tensor [84]. However, neither of the predicted angular momentum scaling laws has been verified in numerical evolutions.

For a perfect fluid with equation of state $p=k \rho$ with $0<k<1 / 9$, precisely one mode that carries angular momentum is unstable, and this mode and the known spherical mode are the only two unstable modes of the spherical critical solution. (Note by comparison that from dimensional analysis one would not expect an uncharged critical solution to have a growing perturbation mode carrying charge.) The presence of two growing modes of the critical solution is expected to give rise to interesting phenomena [100. Near the critical solution, the two growing modes compete. $J$ and $M$ of the final black hole are expected to depend on the distance to the black hole threshold and the angular momentum of the initial data through universal functions of one variable that are similar to "universal scaling functions" in statistical mechanics (see also the end of Sec. 2.7). While they have not yet been computed, these functions can in principle be determined from time evolutions of a single 2-parameter family of initial data, and then determine $J$ and $M$ for all initial data near the black hole threshold and with small angular momentum. They would extend the simple power-law scalings of $J$ and $M$ into a region of initial data space with larger angular momentum.

\subsection{Axisymmetric vacuum gravity}

Abrahams and Evans 1 have numerically investigated black hole formation in axisymmetric vacuum gravity. They write the metric as

$$
d s^{2}=-\alpha^{2} d t^{2}+\phi^{4}\left[e^{2 \eta / 3}\left(d r+\beta^{r} d t\right)^{2}+r^{2} e^{2 \eta / 3}\left(d \theta+\beta^{\theta} d t\right)^{2}+e^{-4 \eta / 3} r^{2} \sin ^{2} \theta d \varphi^{2}\right],
$$

where the lapse $\alpha$, shift components $\beta^{r}$ and $\beta^{\theta}$, and 3-metric coefficients $\phi$ and $\eta$ are functions of $r, t$ and $\theta$. Axisymmetry limits gravitational waves to one polarisation out of two, so that there are as many physical degrees of freedom as in a single wave equation. On the initial slice, $\eta$ and $K_{\theta}^{r}$ are given as free data, and $\phi, K_{r}^{r}$, and $K_{\varphi}^{\varphi}$ are determined by solving the Hamiltonian constraint and the two independent components of the momentum constraint. Afterwards, $\eta, K_{\theta}^{r}, K_{r}^{r}$, and $K_{\varphi}^{\varphi}$ are evolved, and only $\phi$ is obtained by solving the Hamiltonian constraint. $\alpha$ is obtained by solving the maximal slicing condition $K_{i}{ }^{i}=0$ for $\alpha$, and $\beta^{r}$ and $\beta^{\theta}$ are obtained from the time derivatives quasi-isotropic spatial gauge conditions $g_{\theta \theta}=r^{2} g_{r r}$ and $g_{r \theta}=0$.

In order to keep their numerical grid as small as possible, Abrahams and Evans chose their initial data to be mostly ingoing. The two free functions $\eta$ and $K_{\theta}^{r}$ in the initial data were chosen to have the same functional form they would have in a linearised gravitational wave with pure $l=2, m=0$ angular dependence. This ansatz reduced the freedom in the initial data to one free function of advanced time. A specific peaked function was chosen, and only the overall amplitude was varied.

Limited numerical resolution allowed Abrahams and Evans to find black holes with masses only down to 0.2 of the ADM mass. Even this far from criticality, they found power-law scaling of the 
black hole mass, with a critical exponent $\gamma \simeq 0.36$. The black hole mass was determined from the apparent horizon surface area, and the frequencies of the lowest quasi-normal modes of the black hole. There was tentative evidence for scale echoing in the time evolution, with $\Delta \simeq 0.6$, with about three echos seen. Here $\eta$ has the echoing property $\eta\left(e^{\Delta} r, e^{\Delta} t\right)=\eta(r, t)$, and the same echoing property is expected to hold also for $\alpha, \phi, \beta^{r}$ and $r^{-1} \beta^{\theta}$. In a subsequent paper [2], some evidence for universality of the critical solution, echoing period and critical exponent was given in the evolution of a second family of initial data, one in which $\eta=0$ at the initial time. In this family, black hole masses down to 0.06 of the ADM mass were achieved.

It is striking that at 14 years later, these results have not yet been independently verified. An attempt with a 3D numerical relativity code (but axisymmetric initial data), using free evolution in the BSSN formulation with maximal slicing and zero shift, to repeat the results of Abrahams and Evans was not successful [4. The reason could be a combination of rather lower resolution than that of Abrahams and Evans, growing constraint violations, and an inappropriate choice of gauge. (It is now known that BSSN with maximal slicing and zero shift is an ill-posed system 104.)

\subsection{Scalar field}

In 2003, Choptuik, Hirschmann, Liebling and Pretorius reported on numerical evolutions at the black hole threshold of an axisymmetric massless scalar field 52. In axisymmetry with scalar field matter there is no angular momentum and only one polarisation of gravitational waves. The slicing condition is maximal slicing and the spatial gauge, in cylindrical coordinates, is $g_{z z}=g_{\rho \rho}$, $g_{z \rho}=0$, similar to the gauge used by Abrahams and Evans. The Hamiltonian constraint is solved at every time step, and the time derivatives of the spatial gauge conditions are substituted into the momentum constraints to obtain second-order elliptic equations for the two shift components. Thus the evolution is partially constrained. Adaptive mesh-refinement was used in the numerical time evolution.

The initial data were either time-symmetric or approximately ingoing, with the scalar field either symmetric or antisymmetric in $z$. In the symmetric case, even strongly non-spherical data were attracted to the known spherical critical solution for the massless scalar field. Scaling with the known $\gamma$ was observed in the Ricci scalar. However, with sufficiently good fine-tuning to the black hole threshold, the approximately spherical region that approaches the critical solution suffers an $l=2$ (and by ansatz $m=0$ ) instability and splits into two new spherical regions which again approach the critical solution. The spatial separation of the two new centres is related to the smallest length scale that developed prior to the branching. There is evidence that with increasing fine-tuning each of these centres splits again. The antisymmetric initial data cannot approach a single spherical critical solution but the solution splits initially into two approximately spherical regions where the critical solution is approached (up to an overall sign in the scalar field). The separation of these initial two centres is determined by the initial data, but there is evidence that they in turn split.

All this is consistent with the assumption that the spherical critical solution has, besides the known one spherical unstable mode, precisely one further $l=2$ unstable mode. (Without the restriction to axisymmetry, if such a mode exists, it would be 5 -fold degenerate with $m=-2, \ldots, 2$.) This contradicts the calculation of the perturbation spectrum in 146. Choptuik and co-workers do not state with certainty that the mode they see in numerical evolutions is a continuum mode, although they have no indication that it is a numerical artifact. The growth rate of the putative mode is measured to be $\lambda \simeq 0.1-0.4$, which should be compared with the growth rate $\lambda \simeq 2.7$ of the spherical mode and the relatively small decay rate of $\lambda \simeq-0.02$ claimed in [146] for the least damped mode, which is also an $l=2$ mode. We observe that the range of $\tau$ in Figs. 6 and 7 of 52 ] is about 10, and over this range the plot of the amplitude of the $l=2$ perturbation against the 
$\log$-scale coordinate $\tau$ seems equally consistent with linear growth in $\tau$ as with exponential growth. (In the notation of [52, $\tau$ denotes proper time and $\tau_{*}$ the accumulation point of echos, so that the $\log$-scale coordinate $\tau$ used in this review corresponds to $-\ln \left(\tau-\tau_{*}\right)$ in the notation of [52.)

An interesting extension was made in 53 by considering a complex scalar field giving rise to an axisymmetric spacetime with angular momentum. The stress-energy tensor of a complex scalar field $\Psi$ is

$$
T_{a b}=\frac{1}{2}\left(\Psi_{, a} \bar{\Psi}_{, b}+\bar{\Psi}_{, a} \Psi_{, b}-g_{a b} \bar{\Psi}_{, c} \Psi^{, c}\right)
$$

An axisymmetric spacetime with azimuthal Killing vector $\xi$ admits a conserved vector field $T_{a b} \xi^{b}$, so that the total angular momentum

$$
J=\int_{\Sigma} T_{a b} \xi^{a} n^{b} d V
$$

is independent of the Cauchy surface $\Sigma$. With the ansatz

$$
\Psi(\rho, z, t, \varphi)=\Phi(\rho, z, t) e^{i m \varphi},
$$

in adapted coordinates where $\xi=\partial / \partial \varphi$ and with $m$ an integer, the stress-energy tensor becomes axisymmetric and hence compatible with the Einstein equations for an axisymmetric spacetime.

For any $\Sigma$ tangent to $\xi$, in particular a hypersurface $t=$ const., the angular momentum density measured by a normal observer becomes

$$
j=T_{a b} \xi^{a} n^{b}=-\frac{i m}{2}(\Pi \bar{\Phi}-\bar{\Pi} \Phi)=m A^{2} n^{a} \delta_{, a},
$$

where $\Phi=A e^{i \delta}$ with $\delta$ and $A$ real and $\Pi=n^{a} \Phi_{, a}$. By comparison the energy density measured by a normal observer is

$$
\rho=T_{a b} n^{a} n^{b}=\Pi \bar{\Pi}+D_{a} \Phi D^{a} \bar{\Phi},
$$

where $D_{a}$ is the derivative operator projected into $\Sigma$. This means that the ratio of energy density to angular momentum density can be adjusted arbitrarily in the initial data, including zero angular momentum for a $\Phi$ that is real (up to a constant phase). On the other hand, even in the absence of angular momentum a purely real $\Phi$ obeys a wave equation with an explicit $m^{2} / \rho^{2}$ centrifugal term. For the same reason, regular solutions must have $\Phi \sim \rho^{m}$ on the axis, and there are no spherically symmetric solutions. Intuitively speaking, the centrifugal force resisting collapse appears unrelated to the angular momentum component of the stress-energy tensor in a way that differs from what one would expect in rotating fluid collapse or rotating (non-axisymmetric) vacuum collapse.

For all initial data in numerical evolutions, a critical solution is approached that is discretely self-similar with $\log$-scale period $\Delta \simeq 0.42$. (By ansatz this solution is axisymmetric but spherical symmetry is ruled out and so the critical solution cannot be the Choptuik solution.) The same critical solution is approached in particular for initial data with $\Pi=0$ and hence no angular momentum, and initial data where $\Pi=\bar{\Phi}$ and hence with large angular momentum. A scaling exponent of $\gamma \simeq 0.11$ is observed in the Ricci scalar in subcritical evolutions. The critical solution is purely real (up to an initial data-dependent constant phase) and hence has no angular momentum. Only $m=1$ was investigated, but it is plausible that a different critical solution exists for each integer $m$.

Far from the black hole threshold, $J \sim M^{2}$ in the final black hole, but nearer the black hole threshold, $J \sim M^{6}$, where $J$ and $M$ are measured on the apparent horizon when it first forms. $J / M^{2} \rightarrow 0$ is compatible with a non-rotating critical solution.

In the absence of angular momentum, the wave equations for the real and imaginary part of $\Phi$ decouple. Assuming that the background critical solution is purely real with $\delta=0$ and $A \sim 1$, and 
angular momentum is provided by a perturbation with $\delta \sim e^{\lambda \tau}$, one would expect $\rho \sim|\nabla A|^{2} \sim e^{2 \tau}$ and $j \sim A|\nabla \delta| \sim e^{(1+\lambda) \tau}$. Integrating over a region of size $e^{-3 \tau}$ when the black hole forms, we find $M \sim e^{-\tau_{*}}$ and $J \sim e^{(-2+\lambda) \tau_{*}}$. Then $J \sim M^{6}$ would imply $\lambda=-4$.

Lai [134] has studied type I critical phenomena for boson (massive complex scalar field) stars in axisymmetry, the first study of type I in axisymmetry. He finds that the subcritical endstate is a boson star with a large amplitude fundamental mode oscillation.

\subsection{Neutron star collision in axisymmetry}

A first investigation of type I critical collapse in an astrophysically motivated scenario was carried out in [129. The matter is a perfect fluid with "Gamma law" equation of state $P=(\gamma-1) \rho \epsilon$, where $\Gamma \simeq 2$ is a constant, $P$ is the pressure, $\rho$ the rest mass density and $\epsilon$ the internal energy per rest mass (so that $\rho(1+\epsilon)$ is the total energy density). The initial data are constructed with $P=k \rho^{\Gamma}$ for a constant $k$, which corresponds to the "cold" (constant entropy) limit of the Gamma law equation of state. The initial data correspond to two identical stars which have fallen from infinity. (The evolution starts at finite distance, with an initial velocity calculated in the first post-Newtonian approximation). The entire solution is axisymmetric with an additional reflection symmetry that maps one star to the other.

The parameter of the initial data that is varied is the mass of the two stars. Supercritical data form a single black hole, while subcritical data form a single star. The diagnostics given are plots against time of the lapse and the Ricci scalar at the symmetry centre of the spacetime, and of outgoing $l=2$ gravitational waves. Collapse of the lapse and blowup of the Ricci scalar are taken as indications of black hole formation. The limited numerical evidence is compatible with type I critical phenomena, with the putative critical solution showing oscillations with the same period in the lapse and Ricci scalar. For the critical solution to be exactly time-periodic, it would have to be spherical in order to not lose energy through gravitational waves, and there is some evidence that indeed it does not radiate gravitational waves.

Other one-parameter families of initial data were obtained by fixing the mass of the stars in the first family very near its critical value and then varying one of the following: the initial separation, the initial speed, and $\Gamma$. For all approximately the same scaling law for the survival time of the critical solution was found. However, as all these initial data are very close together, this only confirms the validity of the general perturbation theory explanation of critical phenomena, but does not provide evidence of universality.

A key question that has not been answered is how shock heating affects the standard critical collapse scenario. A priori the existence of a universal spherical critical solution is unlikely in the presence of shock heating as there is no dynamical mechanism to arrive at a universal spherical temperature profile, in contrast to non-dissipative matter models or vacuum gravity where all equations are wave-like.

\subsection{Black hole collisions}

Interesting numerical evidence for critical phenomena in the glancing collision of two black holes has been found by Pretorius [167. He evolved initial data for two equal mass nonrotating black holes, with a reflection symmetry through the orbital plane, parameterised by their relative boost at constant impact parameter. The threshold in initial data space is between data which merge immmediately and those which do not (although they will merge later for initial data which are bound). The critical solution is a circular orbit that loses $1-1.5 \%$ of total energy per orbit through radiation. On both sides of the threshold, the number of orbits scales as

$$
n \simeq-\gamma \ln \left|p-p_{*}\right|,
$$


for $\gamma \simeq 0.31-0.38$. The simulations are currently limited by numerical accuracy to $n<5$ and $\Delta E / E<0.08$ but Pretorius conjectures that the total energy loss and hence the number of orbits is limited only by the irreducible mass of the initial data, a much larger number. In particular, he speculates that for highly boosted initial data such that the total energy of the initial data is dominated by kinetic energy, almost all the energy can be converted into gravitational radiation.

The standard dynamical systems picture of critical collapse, with the critical solution an attractor in the threshold hypersurface, appears to be consistent with these observation. Pretorius compares his data with the unstable circular geodesics in the spacetime of the hypothetical rotating black hole that would result if merger occurred promptly. These give orbital periods, and their linear perturbations give a critical exponent, in rough agreement with the numerical values for the full black hole collision.

Pretorius does not comment on the nature of the critical solution, but because of the mass loss through gravitational radiation it cannot be strictly stationary. The mass loss would be compatible with self-similarity, with a helical homothetic vector field, but exact self-similarity would be compatible with the presence of black holes only in the infinite boost limit. Therefore the critical solution is likely to be more complicated, and can perhaps be written as an expansion with an exactly stationary or homothetic spacetime as the leading term.

Pretorius also speculates that these phenomena generalise to generic initial data with unequal masses and black hole spins which are not aligned. This seems uncertain, given the claim by Levin [139] that the threshold of immediate merger is fractal if the spins are not aligned, and that the system is therefore chaotic. However, Levin's analysis is based on a 2nd order post-Newtonian approximation to general relativity, in which there is no radiation reaction, while the rapid energy loss observed here may suppress chaos. Nevertheless, the phase space is much bigger when the orbit is not confined to an orbital plane, and so the critical solution observed here may not be an attractor in the full critical surface.

\section{Thanks}

We would like to thank David Garfinkle for a critical reading of the manuscript and Louisiana State University for hospitality while this work was begun. JMM was supported by the I3P framework of CSIC and the European Social Fund, and by the Spanish MEC Project FIS2005-05736-C03-02.

\section{References}

[1] Abrahams, A.M., and Evans, C.R., "Critical behavior and scaling in vacuum axisymmetric gravitational collapse", Phys. Rev. Lett., 70, 2980-2983, (1993).

[2] Abrahams, A.M., and Evans, C.R., "Universality in axisymmetric vacuum collapse", Phys. Rev. D, 49, 3998-4003, (1994).

[3] Aichelburg, P.C., Bizoń, P., and Tabor, Z., "Bifurcation and fine structure phenomena in critical collapse of a self-gravitating $\sigma$-field", Class. Quant. Grav., 23, S299-S306, (2006). Related online version (cited on 15 June 2007):

http://arXiv.org/abs/gr-qc/0512136.

[4] Alcubierre, M., Allen, G., Bruegmann, B., Lanfermann, G., Seidel, E., Suen, W.-M., and Tobias, M., "Gravitational collapse of gravitational waves in 3D numerical relativity", Phys. Rev. D, 61, 041501, (2000). Related online version (cited on 1 February 2003): http://arXiv.org/abs/gr-qc/9904013 
[5] Álvarez-Gaumé, L., Gómez, C., and Vázquez-Mozo, M.A., "Scaling Phenomena in Gravity from QCD", Phys. Lett. B, 649, 478-482, (2007). Related online version (cited on 15 June 2007): http://arXiv.org/abs/hep-th/0611312

[6] Andreasson, H., and Rein, G., "A numerical investigation of the stability of steady states and critical phenomena for the spherically symmetric Einstein-Vlasov system", Class. Quant. Grav., 23, 3659-3678, (2006). Related online version (cited on 15 June 2007): http://arXiv.org/abs/gr-qc/0601112.

[7] Ayal, S., and Piran, T., "Spherical collapse of a mass-less scalar field with semi-classical corrections", Phys. Rev. D, 56, 4768-4774, (1997). Related online version (cited on 10 December 1999): http://arXiv.org/abs/gr-qc/9704027

[8] Bartnik, R., and McKinnon, J., "Particle-like solutions of the Einstein-Yang-Mills equations", Phys. Rev. Lett., 61, 141-144, (1988).

[9] Birmingham, D., "Choptuik scaling and quasinormal modes in the AdS/CFT correspondence", Phys. Rev. D, 64, 064024, (2001). Related online version (cited on 1 February 2003): http://arXiv.org/abs/hep-th/0101194.

[10] Birmingham, D., and Sen, S., "Gott time machines, BTZ black hole formation, and Choptuik scaling", Phys. Rev. Lett., 84, 1074, (2000). Related online version (cited on 1 February 2003): http://arXiv.org/abs/hep-th/9908150.

[11] Birukou, M., Husain, V., Kunstatter, G., Vaz, E., and Olivier, M., "Scalar field collapse in any dimension", Physical Rev. D, 65, 104036, (2002). Related online version (cited on 1 February 2003): http://arXiv.org/abs/gr-qc/0201026

[12] Bizoń, P., "Colored black holes", Phys. Rev. Lett., 64, 2844-2847, (1990).

[13] Bizoń, P., "How to Make a Tiny Black Hole?", Acta Cosmologica, 22, 81, (1996). Related online version (cited on 10 December 1999): http://arXiv.org/abs/gr-qc/9606060

[14] Bizoń, P., "Equivariant self-similar wave maps from Minkowski spacetime into 3-sphere", Commun. Math. Phys., 215, 45, (2000). Related online version (cited on 15 June 2007): http://arXiv.org/abs/math-ph/9910026.

[15] Bizoń, P., and Chmaj, T., "Formation and critical collapse of Skyrmions", Phys. Rev. D, 58, 041501-1-4, (1998). Related online version (cited on 10 December 1999): http://arXiv.org/abs/gr-qc/9801012.

[16] Bizoń, P., and Chmaj, T., "Remark on formation of colored black holes via fine-tuning", Phys. Rev. D, 61, 067501-1-2, (June, 2000). Related online version (cited on 10 December 1999): http://arXiv.org/abs/gr-qc/9906070

[17] Bizoń, P., Chmaj, T., Rostworowski, A., Schmidt, B.G., and Tabor, Z., "On vacuum gravitational collapse in nine dimensions", Phys. Rev. D, 72, 121502, (2005). Related online version (cited on 15 June 2007): http://arXiv.org/abs/gr-qc/0511064 
[18] Bizoń, P., Chmaj, T., and Schmidt, B.G., "Critical behavior in vacuum gravitational collapse in 4+1 dimensions", Phys. Rev. Lett., 95, 071102, (2005). Related online version (cited on 15 June 2007): http://arXiv.org/abs/gr-qc/0506074.

[19] Bizoń, P., Chmaj, T., and Schmidt, B.G., "Codimension-two critical behavior in vacuum gravitational collapse", Phys. Rev. Lett., 97, 131101, (2006). Related online version (cited on 15 June 2007):

http://arXiv.org/abs/gr-qc/0608102.

[20] Bizoń, P., Chmaj, T., and Tabor, Z., "On Equivalence of Critical Collapse of Non-Abelian Field", Phys. Rev. D, 59, 104003, (1999). Related online version (cited on 10 December 1999):

http://arXiv.org/abs/gr-qc/9901039

[21] Bizoń, P., Chmaj, T., and Tabor, Z., "Dispersion and collapse of wave maps", Nonlinearity, 13, 1411, (2000). Related online version (cited on 1 February 2003): http://arXiv.org/abs/math-ph/9912009.

[22] Bizoń, P., Chmaj, T., and Tabor, Z., "Formation of singularities for equivariant $2+1$ dimensional wave maps into the 2-sphere", Nonlinearity, 14, 1041, (2001).

[23] Bizoń, P., Szybka, S., and Wassermann, A., "Periodic self-similar wave maps coupled to gravity", Phys. Rev. D, 69, 064014, (2004). Related online version (cited on 15 June 2007): http://arXiv.org/abs/gr-qc/0310038

[24] Bizoń, P., and Tabor, Z., "On blowup for Yang-Mills fields", Phys. Rev. D, 64, 121701, (2001). Related online version (cited on 1 February 2003): http://arXiv.org/abs/math-ph/0105016.

[25] Bizoń, P., and Wassermann, A., "Self-similar spherically symmetric wave maps coupled to gravity", Phys. Rev. D, 62, 084031, (2000). Related online version (cited on 1 February 2003): http://arXiv.org/abs/gr-qc/0006034

[26] Bizoń, P., and Wassermann, A., "On the existence of self-similar spherically symmetric wave maps coupled to gravity", Class. Quant. Grav., 19, 3309-3322, (2002). Related online version (cited on 15 June 2007): http://arXiv.org/abs/gr-qc/0201046

[27] Bland, J., and Kunstatter, G., "The 5-D Choptuik critical exponent and holography", (2007). Related online version (cited on 15 June 2007): http://arXiv.org/abs/hep-th/0702226.

[28] Bland, J., Preston, B., Becker, M., Kunstatter, G., and Husain, V., "Dimension-Dependence of the Critical Exponent in Spherically Symmetric Gravitational Collapse", (2005). Related online version (cited on 15 June 2007): http://arXiv.org/abs/gr-qc/0507088

[29] Bose, S., Parker, L., and Peleg, Y., "Predictability and semiclassical approximation at the onset of black hole formation", Phys. Rev. D, 54, 7490-7505, (1996). Related online version (cited on 10 December 1999):

http://arXiv.org/abs/hep-th/9606152. 
[30] Brady, P., Choptuik, M.W., Gundlach, C., and Neilsen, D., "Black-hole threshold solutions in stiff fluid collapse", Class. Quant. Grav., 19, 6359, (2002). Related online version (cited on 1 February 2003):

http://arXiv.org/abs/gr-qc/0207096

[31] Brady, P.R., "Does scalar field collapse produce 'zero mass' black holes?", Class. Quant. Grav., 11, 1255, (1994). Related online version (cited on 10 December 1999): http://arXiv.org/abs/gr-qc/9402023

[32] Brady, P.R., and Cai, M.J., "Critical phenomena in gravitational collapse", in Piran, T., ed., The Eighth Marcel Grossmann Meeting on Recent Developments in Theoretical and Experimental General Relativity, Gravitation and Relativistic Field Theories, Proceedings of the meeting held at the Hebrew University of Jerusalem, June 22-27, 1997, 689-704, (World Scientific, Singapore, 1999). Related online version (cited on 10 December 1999): http://arXiv.org/abs/gr-qc/9812071

[33] Brady, P.R., Chambers, C.M., and Gonçalves, S.M.C.V., "Phases of massive scalar field collapse", Phys. Rev. D, 56, 6057-6061, (1997). Related online version (cited on 10 December 1999):

http://arXiv.org/abs/gr-qc/9709014

[34] Brady, P.R., and Ottewill, A.C., "Quantum corrections to critical phenomena in gravitational collapse", Phys. Rev. D, 58, 024006, (1998). Related online version (cited on 10 December 1999):

http://arXiv.org/abs/gr-qc/9804058.

[35] Burko, L.M., "Black hole singularities: a new critical phenomenon", Phys. Rev. Lett., 90, 121101, (2003). Related online version (cited on 15 June 2007):

http://arXiv.org/abs/gr-qc/0209084.

[36] Cahill, M.E., and Taub, A.H., "Spherically symmetric similarity solutions of the Einstein equations for a perfect fluid", Commun. Math. Phys., 21, 1, (1971).

[37] Carlip, S., "The (2+1)-dimensional black hole", Class. Quant. Grav., 12, 2853, (1995). Related online version (cited on 1 February 2003):

http://arXiv.org/abs/gr-qc/9506079

[38] Carr, B.J., and Coley, A.A., "A complete classification of spherically symmetric perfect fluid similarity solutions", Phys. Rev. D, 62, 044023, (2000). Related online version (cited on 1 February 2003):

http://arXiv.org/abs/gr-qc/9901050

[39] Carr, B.J., Coley, A.A., Goliath, M., Nilsson, U.S., and Uggla, C., "Critical phenomena and a new class of self-similar spherically symmetric perfect-fluid solutions", Phys. Rev. D, 61, 081502-1-5, (2000). Related online version (cited on 10 December 1999):

http://arXiv.org/abs/gr-qc/9901031.

[40] Carr, B.J., and Gundlach, C., "Spacetime structure of self-similar spherically symmetric perfect fluid solutions", Phys. Rev. D, 67, 024035, (2003). Related online version (cited on 1 February 2003):

http://arXiv.org/abs/gr-qc/0209092

[41] Cavaglià, M., Clément, G., and Fabbri, A., "Approximately self-similar critical collapse in 2+1 dimensions", Phys. Rev. D, 70, 044010, (2004). Related online version (cited on 15 June 
2007):

http://arXiv.org/abs/gr-qc/0404033

[42] Caveny, S.A., and Matzner, R.A., "Adaptive event horizon tracking and critical phenomena in binary black hole coalescence", Phys. Rev. D, 68, 104003, (2003). Related online version (cited on 15 June 2007): http://arXiv.org/abs/gr-qc/0303109

[43] Chiba, T., and Siino, M., "Disappearance of black hole criticality in semiclassical general relativity", Mod. Phys. Lett. A, 12, 709, (1997).

[44] Choptuik, M.W., personal communication.

[45] Choptuik, M.W., "Critical' behavior in massless scalar field collapse", in d'Inverno, R., ed., Approaches to Numerical Relativity, Proceedings of the International Workshop on Numerical Relativity, Southampton, December 1991, 202, (Cambridge University Press, Cambridge, U.K.; New York, U.S.A., 1992).

[46] Choptuik, M.W., "Universality and scaling in gravitational collapse of a massless scalar field", Phys. Rev. Lett., 70(1), 9-12, (1993).

[47] Choptuik, M.W., "Critical behavior in scalar field collapse", in Hobill, D., Burd, A., and Coley, A., eds., Deterministic Chaos in General Relativity, Proceedings of a NATO Advanced Research Workshop on Deterministic Chaos in General Relativity, held July 25-30, 1993, in Kananaskis, Alberta, Canada, 155, (Plenum Press, New York, U.S.A., 1994).

[48] Choptuik, M.W., "The (Unstable) Threshold of Black Hole Formation", in Dadhich, N., and Narlikar, J.V., eds., Gravitation and Relativity: At the Turn of the Millenium, Proceedings of the 15th International Conference on General Relativity and Gravitation (GR-15), held at IUCAA, Pune, India, December 16-21, 1997, 67-85, (IUCAA, Pune, India, 1998). Related online version (cited on 10 December 1999):

http://arXiv.org/abs/gr-qc/9803075

[49] Choptuik, M.W., "Critical behavior in gravitational collapse", Prog. Theor. Phys. Suppl., 136, 353, (1999).

[50] Choptuik, M.W., Chmaj, T., and Bizoń, P., "Critical Behavior in Gravitational Collapse of a Yang-Mills Field", Phys. Rev. Lett., 77, 424-427, (1996). Related online version (cited on 10 December 1999): http://arXiv.org/abs/gr-qc/9603051

[51] Choptuik, M.W., Hirschmann, E.W., and Liebling, S.L., "Instability of an 'Approximate black hole", Phys. Rev. D, 55, 6014-6018, (1997). Related online version (cited on 10 December 1999): http://arXiv.org/abs/gr-qc/9701011.

[52] Choptuik, M.W., Hirschmann, E.W., Liebling, S.L., and Pretorius, F., "Critical Collapse of the Massless Scalar Field in Axisymmetry", Phys. Rev. D, 68, 044007, (2003). Related online version (cited on 15 June 2007): http://arXiv.org/abs/gr-qc

[53] Choptuik, M.W., Hirschmann, E.W., Liebling, S.L., and Pretorius, F., "Critical Collapse of a Complex Scalar Field with Angular Momentum", Phys. Rev. Lett, 93, 131101, (2004). Related online version (cited on 15 June 2007): http://arXiv.org/abs/gr-qc/0405101. 
[54] Choptuik, M.W., Hirschmann, E.W., and Marsa, R.L., "New critical behavior in EinsteinYang-Mills collapse", Phys. Rev. D, 60, 124011-1-9, (1999). Related online version (cited on 10 December 1999): http://arXiv.org/abs/gr-qc/9903081.

[55] Christodoulou, D., "Violation of cosmic censorship in the gravitational collapse of a dust cloud", Commun. Math. Phys., 93, 17, (1984).

[56] Christodoulou, D., "The problem of a self-gravitating scalar field", Commun. Math. Phys., 105(3), 337-361, (1986).

[57] Christodoulou, D., "A mathematical theory of gravitational collapse", Commun. Math. Phys., 109(4), 613-647, (1987).

[58] Christodoulou, D., "The formation of black holes and singularities in spherically symmetric gravitational collapse", Commun. Pure Appl. Math., 44, 339-373, (1991).

[59] Christodoulou, D., "Bounded variation solutions of the spherically symmetric Einstein-scalar field equations", Commun. Pure Appl. Math., 46, 1131-1220, (1993).

[60] Christodoulou, D., "Examples of naked singularity formation in the gravitational collapse of a scalar field", Ann. Math., 140, 607, (1994).

[61] Christodoulou, D., "The instability of naked singularities in the gravitational collapse of a scalar field", Ann. Math. (2), 149, 183-217, (1999).

[62] Clement, G., and Fabbri, A., "Analytical treatment of critical collapse in $2+1$ dimensional AdS spacetime: a toy model", Class. Quant. Grav., 18, 3665, (2001). Related online version (cited on 1 February 2003):

http://arXiv.org/abs/gr-qc/0101073.

[63] Clement, G., and Fabbri, A., "Critical collapse in 2+1 dimensional AdS spacetime: quasiCSS solutions and linear perturbations", Nucl. Phys. B, 269, 269, (2002). Related online version (cited on 1 February 2003):

http://arXiv.org/abs/gr-qc/0109002.

[64] Clement, G., and Hayward, S.A., "Comment on 'An extreme critical space-time: echoing and black-hole perturbations"', Class. Quant. Grav., 18, 4715, (2001). Related online version (cited on 1 February 2003):

http://arXiv.org/abs/gr-qc/0108024.

[65] Donninger, R., and Aichelburg, P.C., "A note on the eigenvalues for equivariant maps of the $S U(2)$ sigma-model", (2006). Related online version (cited on 15 June 2007):

http://arXiv.org/abs/math-ph/0601019.

[66] Donninger, R., and Aichelburg, P.C., "On the mode stability of a self-similar wave map", (2007). Related online version (cited on 15 June 2007):

http://arXiv.org/abs/math-ph/0702025.

[67] Eardley, D.M., Hirschmann, E.W., and Horne, J.H., " $S$ duality at the black hole threshold in gravitational collapse", Phys. Rev. D, 52, R5397-R5401, (1995). Related online version (cited on 10 December 1999):

http://arXiv.org/abs/gr-qc/9505041 
[68] Eardley, D.M., and Moncrief, V., "The global existence of Yang-Mills-Higgs fields in 4dimensional Minkowski space I. Local existence and smoothness properties", Comm. Math. Phys., 83, 171-191, (1982).

[69] Evans, C.R., and Coleman, C.S., "Critical Phenomena and Self-Similarity in the Gravitational Collapse of Radiation Fluid", Phys. Rev. Lett., 72, 1782-1785, (1994).

[70] Frolov, A.V., "Perturbations and critical behavior in the self-similar gravitational collapse of a massless scalar field", Phys. Rev. D, 56, 6433-6438, (1997). Related online version (cited on 10 December 1999): http://arXiv.org/abs/gr-qc/9704040.

[71] Frolov, A.V., "Critical Collapse Beyond Spherical Symmetry: General Perturbations of the Roberts Solution", Phys. Rev. D, 59, 104011, (1999). Related online version (cited on 10 December 1999): http://arXiv.org/abs/gr-qc/9811001.

[72] Frolov, A.V., "Self-Similar Collapse of Scalar Field in Higher Dimensions", Class. Quant. Grav., 16, 407-417, (1999). Related online version (cited on 15 June 2007): http://arXiv.org/abs/gr-qc/9806112.

[73] Frolov, A.V., "Continuous self-similarity breaking in critical collapse", Phys. Rev. D, 61, 084006-1-14, (2000). Related online version (cited on 10 December 1999): http://arXiv.org/abs/gr-qc/9908046.

[74] Frolov, A.V., and Pen, Ue-Li, "The naked singularity in the global structure of critical collapse spacetimes", Phys. Rev. D, 68, 124024, (2003). Related online version (cited on 15 June 2007): http://arXiv.org/abs/gr-qc/0307081

[75] Frolov, V.P., "Merger transitions in brane-black-hole systems: criticality, scaling and selfsimilarity", Phys. Rev. D, 74, 044006, (2006). Related online version (cited on 15 June 2007): http://arXiv.org/abs/gr-qc/0604114.

[76] Frolov, V.P., Larsen, A.L., and Christensen, M., "Domain wall interacting with a black hole: A new example of critical phenomena", Phys. Rev. D, 59, 125008, (1999). Related online version (cited on 15 June 2007): http://arXiv.org/abs/hep-th/9811148

[77] Garfinkle, D., "Choptuik scaling in null coordinates", Phys. Rev. D, 51(10), 5558-5561, (1995). Related online version (cited on 10 December 1999): http://arXiv.org/abs/gr-qc/9412008.

[78] Garfinkle, D., "Choptuik scaling and the scale invariance of Einstein's equation", Phys. Rev. $D, \mathbf{5 6}, 3169-3173,(1997)$. Related online version (cited on 10 December 1999): http://arXiv.org/abs/gr-qc/9612015.

[79] Garfinkle, D., "An exact solution for $2+1$ dimensional critical collapse", Phys. Rev. D, 63, 044007, (2001). Related online version (cited on 1 February 2003): http://arXiv.org/abs/gr-qc/0008023.

[80] Garfinkle, D., Cutler, C., and Duncan, G.C., "Choptuik scaling in six dimensions", Phys. Rev. D, 60, 104007-1-5, (1999). Related online version (cited on 10 December 1999): http://arXiv.org/abs/gr-qc/9908044. 
[81] Garfinkle, D., and Duncan, G.C., "Scaling of curvature in sub-critical gravitational collapse", Phys. Rev. D, 58, 064024, (1998). Related online version (cited on 10 December 1999): http://arXiv.org/abs/gr-qc/9802061.

[82] Garfinkle, D., and Gundlach, C., "Symmetry-seeking spacetime coordinates", Class. Quant. Grav., 16, 4111-4123, (1999). Related online version (cited on 10 December 1999): http://arXiv.org/abs/gr-qc/9908016.

[83] Garfinkle, D., and Gundlach, C., "Perturbations of an exact solution for 2+1-dimensional critical collapse", Phys. Rev. D, 66, 044015, (2002). Related online version (cited on 1 February 2003): http://arXiv.org/abs/gr-qc/0205107

[84] Garfinkle, D., Gundlach, C., and Martín-García, J.M., "Angular momentum near the black hole threshold in scalar field collapse", Phys. Rev. D, 59, 104012, (1999). Related online version (cited on 10 December 1999): http://arXiv.org/abs/gr-qc/9811004.

[85] Garfinkle, D., and Isenberg, J., "Numerical studies of the behavior of Ricci flow", Contemporary Mathematics, 367, 103, (2005). Related online version (cited on 15 June 2007): http://arXiv.org/abs/math/0306129

[86] Garfinkle, D., Mann, R., and Vuille, C., "Critical collapse of a massive vector field", Phys. Rev. D, 68, 064015, (2003). Related online version (cited on 15 June 2007): http://arXiv.org/abs/gr-qc/0305014.

[87] Garfinkle, D., and Meyer, K., "Scale invariance and critical gravitational collapse", Phys. Rev. D, 59, 064003, (1999). Related online version (cited on 10 December 1999): http://arXiv.org/abs/gr-qc/9806052.

[88] Giddings, S.B., "Quantum mechanics of black holes", (December, 1994). Related online version (cited on 10 December 1999): http://arXiv.org/abs/hep-th/9412138. Lectures presented at the 1994 Trieste Summer School in High Energy Physics and Cosmology.

[89] Goode, S.W., Coley, A.A., and Wainwright, J., "The isotropic singularity in cosmology", Class. Quant. Grav., 9, 445, (1992).

[90] Green, A.M., and Liddle, A.R., "Critical collapse and the primordial black hole initial mass function", Phys. Rev. D, 60, 063509, (1999). Related online version (cited on 1 February 2003): http://arXiv.org/abs/astro-ph/9901268v2

[91] Gundlach, C., "The Choptuik Spacetime as an Eigenvalue Problem", Phys. Rev. Lett., 75, 3214-3217, (1995). Related online version (cited on 10 December 1999): http://arXiv.org/abs/gr-qc/9507054

[92] Gundlach, C., "Critical phenomena in gravitational collapse", in Chruściel, P.T., ed., Mathematics of Gravitation, Part I: Lorentzian Geometry and Einstein Equations, Proceedings of the Workshop on Mathematical Aspects of Theories of Gravitation, held in Warsaw, Poland, February 29 - March 30, 1996, vol. 41 of Banach Center Publications, 143-152, (Polish Academy of Sciences, Institute of Mathematics, Warsaw, Poland, 1997). Related online version (cited on 10 December 1999): http://arXiv.org/abs/gr-qc/9606023. 
[93] Gundlach, C., "Echoing and scaling in Einstein-Yang-Mills critical collapse", Phys. Rev. D, 55, 6002-6013, (1997). Related online version (cited on 10 December 1999): http://arXiv.org/abs/gr-qc/9610069.

[94] Gundlach, C., "Understanding critical collapse of a scalar field", Phys. Rev. D, 55, 695-713, (1997). Related online version (cited on 10 December 1999): http://arXiv.org/abs/gr-qc/9507054.

[95] Gundlach, C., "Angular momentum at the black hole threshold", Phys. Rev. D, 57, 70807083, (1998). Related online version (cited on 10 December 1999): http://arXiv.org/abs/gr-qc/9711079.

[96] Gundlach, C., "Critical phenomena in gravitational collapse", Adv. Theor. Math. Phys., 2, 1-49, (1998). Related online version (cited on 10 December 1999): http://arXiv.org/abs/gr-qc/9712084.

[97] Gundlach, C., "Nonspherical perturbations of critical collapse and cosmic censorship", Phys. Rev. D, 57, 7075-7079, (1998). Related online version (cited on 10 December 1999): http://arXiv.org/abs/gr-qc/9710066.

[98] Gundlach, C., "Critical Phenomena in Gravitational Collapse", Living Rev. Rel., 2, 4, (1999). Related online version (cited on 1 February 2003):

http://www.livingreview.org

[99] Gundlach, C., "Critical gravitational collapse of a perfect fluid with $p=k \rho$ : Nonspherical perturbations", Phys. Rev. D, 65, 084021-1-22, (2002). Related online version (cited on 10 December 1999):

http://arXiv.org/abs/gr-qc/9906124v2.

[100] Gundlach, C., "Critical gravitational collapse with angular momentum: from critical exponents to universal scaling functions", Phys. Rev. D, 65, 064019, (2002). Related online version (cited on 1 February 2003): http://arXiv.org/abs/gr-qc/0110049.

[101] Gundlach, C., "Critical Phenomena in Gravitational Collapse", Phys. Rep., 376, 339, (2003). Related online version (cited on 1 February 2003): http://arXiv.org/abs/gr-qc/0210101.

[102] Gundlach, C., and Martín-García, J.M., "Charge scaling and universality in critical collapse", Phys. Rev. D, 54, 7353-7360, (1996). Related online version (cited on 10 December 1999): http://arXiv.org/abs/gr-qc/9606072.

[103] Gundlach, C., and Martín-García, J.M., "Kinematics of discretely self-similar spherically symmetric spacetimes", Phys. Rev. D, 68, 064019, (2003). Related online version (cited on 15 June 2007):

http://arXiv.org/abs/gr-qc/0306001

[104] Gundlach, C., and Martín-García, J.M., "Well-posedness of formulations of the Einstein equations with dynamical lapse and shift conditions", Phys. Rev. D, 74, 024016, (2006). Related online version (cited on 15 June 2007): http://arXiv.org/abs/gr-qc/0604035

[105] Gundlach, C., Price, R.H., and Pullin, J., "Late-time behavior of stellar collapse and explosions. II. Nonlinear evolution", Phys. Rev. D, 49, 890-899, (1994). Related online version (cited on 10 December 1999): http://arXiv.org/abs/gr-qc/9307010 
[106] Hamadé, R.S., Horne, J.H., and Stewart, J.M., "Continuous self-similarity and S-duality", Class. Quant. Grav., 13, 2241-2253, (1996). Related online version (cited on 10 December 1999): http://arXiv.org/abs/gr-qc/9511024

[107] Hamadé, R.S., and Stewart, J.M., "The spherically symmetric collapse of a massless scalar field", Class. Quant. Grav., 13, 497-512, (1996). Related online version (cited on 10 December 1999): http://arXiv.org/abs/gr-qc/9506044

[108] Hara, T., Koike, T., and Adachi, S., "Renormalization group and critical behavior in gravitational collapse", (July, 1996). Related online version (cited on 10 December 1999): http://arXiv.org/abs/gr-qc/9607010.

[109] Harada, T., "Final fate of the spherically symmetric collapse of a perfect fluid", Phys. Rev. $D, \mathbf{5 8}, 104015,(1998)$. Related online version (cited on 1 February 2003): http://arXiv.org/abs/gr-qc/9807038

[110] Harada, T., "Stability criterion for self-similar solutions with perfect fluids in general relativity", Class. Quant. Grav., 18, 4549, (2001). Related online version (cited on 1 February 2003): http://arXiv.org/abs/gr-qc/0109042v2.

[111] Harada, T., Hideki, M., and Semelin, B., "Criticality and convergence in Newtonian collapse", Phys. Rev. D, 67084003, 084003, (2003). Related online version (cited on 15 June 2007): http://arXiv.org/abs/gr-qc/0210027

[112] Harada, T., and Maeda, H., "Convergence to a self-similar solution in general relativistic gravitational collapse", Phys. Rev. D, 63, 084022, (2001). Related online version (cited on 1 February 2003): http://arXiv.org/abs/gr-qc/0101064

[113] Harada, T., and Maeda, H., "Critical phenomena in Newtonian gravity", Phys. Rev. D, 64, 124024, (2001). Related online version (cited on 1 February 2003): http://arXiv.org/abs/gr-qc/0109095.

[114] Hawke, I., and Stewart, J.M., "The dynamics of primordial black-hole formation", Class. Quant. Grav., 19, 3687-3707, (2002).

[115] Hawley, S.H., and Choptuik, M.W., "Boson stars driven to the brink of black hole formation", Phys. Rev. D, 62, 104024, (2000). Related online version (cited on 1 February 2003): http://arXiv.org/abs/gr-qc/0007039.

[116] Hayward, S.A., "An extreme critical space-time: echoing and black-hole perturbations", Class. Quant. Grav., 17, 4021, (2000). Related online version (cited on 1 February 2003): http://arXiv.org/abs/gr-qc/0004038.

[117] Hirschmann, E.W., and Eardley, D.M., "Critical exponents and stability at the black hole threshold for a complex scalar field", Phys. Rev. D, 52, 5850-5856, (1995). Related online version (cited on 10 December 1999): http://arXiv.org/abs/gr-qc/9506078 
[118] Hirschmann, E.W., and Eardley, D.M., "Universal scaling and echoing in gravitational collapse of a complex scalar field", Phys. Rev. D, 51, 4198-4207, (1995). Related online version (cited on 10 December 1999): http://arXiv.org/abs/gr-qc/9412066

[119] Hirschmann, E.W., and Eardley, D.M., "Criticality and bifurcation in the gravitational collapse of a self-coupled scalar field", Phys. Rev. D, 56, 4696-4705, (1997). Related online version (cited on 10 December 1999):

http://arXiv.org/abs/gr-qc/9511052

[120] Hirschmann, E.W., Wang, A., and Wu, Y., "Collapse of a Scalar Field in 2+1 Gravity", Class. Quant. Grav., 21, 1791-1824, (2004). Related online version (cited on 1 February 2003):

http://arXiv.org/abs/gr-qc/0207121.

[121] Hod, S., and Piran, T., "Critical behaviour and universality in gravitational collapse of a charged scalar field", Phys. Rev. D, 55, 3485-3496, (1997). Related online version (cited on 10 December 1999): http://arXiv.org/abs/gr-qc/9606093

[122] Hod, S., and Piran, T., "Fine-structure of Choptuik's mass-scaling relation", Phys. Rev. D, 55, 440-442, (1997). Related online version (cited on 10 December 1999): http://arXiv.org/abs/gr-qc/9606087.

[123] Honda, E.P., and Choptuik, M.W., "Fine structure of oscillons in the spherically symmetric $\phi^{4}$ Klein-Gordon model", Phys. Rev. D, 65, 084037, (2002). Related online version (cited on 1 February 2003): http://arXiv.org/abs/hep-ph/0110065

[124] Horne, J., "Critical behavior in black hole collapse", Matters of Gravity, 7, 14, (1996). Related online version (cited on 10 December 1999): http://arXiv.org/abs/gr-qc/9602001.

[125] Horowitz, G.T., and Hubeny, V.E., "Quasinormal Modes of AdS Black Holes and the Approach to Thermal Equilibrium", Phys. Rev. D, 62, 024027, (2000). Related online version (cited on 1 February 2003): http://arXiv.org/abs/hep-th/9909056.

[126] Husa, S., Lechner, C., Pürrer, M., Thornburg, J., and Aichelburg, P.C., "Type II critical collapse of a self-gravitating nonlinear $\sigma$-model", Phys. Rev. D, 62, 104007, (2000). Related online version (cited on 1 February 2003): http://arXiv.org/abs/gr-qc/0002067.

[127] Husain, V., Kunstatter, G., Preston, B., and Birukou, M., "Anti-deSitter gravitational collapse", Class. Quant. Grav., 20, L23-L30, (2003). Related online version (cited on 1 February 2003): http://arXiv.org/abs/gr-qc/02010011.

[128] Husain, V., and Olivier, M., "Scalar field collapse in three-dimensional AdS spacetime", Class. Quant. Grav., 18, L1, (2001). Related online version (cited on 1 February 2003): http://arXiv.org/abs/gr-qc/0008060.

[129] Jin, K.-J., and Suen, W.-M., "Critical Phenomena in Head-on Collisions of Neutron Stars", Phys. Rev. Lett., 98, 131101, (2007). Related online version (cited on 15 June 2007): http://arXiv.org/abs/gr-qc/0603094. 
[130] Kiem, Y., "Phase transition in spherically symmetric gravitational collapse of a massless scalar field", arxiv, (1994). Related online version (cited on 10 December 1999):

http://arXiv.org/abs/hep-th/9407100.

[131] Koike, T., Hara, T., and Adachi, S., "Critical Behavior in Gravitational Collapse of Radiation Fluid: A Renormalization Group (Linear Perturbation) Analysis", Phys. Rev. Lett., 74, 5170-5173, (1995). Related online version (cited on 10 December 1999): http://arXiv.org/abs/gr-qc/9503007.

[132] Koike, T., Hara, T., and Adachi, S., "Critical behavior in gravitational collapse of a perfect fluid", Phys. Rev. D, 59, 104008, (1999).

[133] Kol, B., "Choptuik Scaling and The Merger Transition", (2005). Related online version (cited on 15 June 2007): http://arXiv.org/abs/hep-th/0502034

[134] Lai, Chi-Wai, A Numerical Study of Boson Stars, (University of British Columbia, Vancouver, 2004). Related online version (cited on 15 June 2007): http://arXiv.org/abs/gr-qc/0410040.

[135] Lai, Chi-Wai, and Choptuik, M.W., "Final fate of subcritical evolutions of boson stars", (2007). Related online version (cited on 27 November 2007): http://arXiv.org/abs/0709.0324.

[136] Lavrelashvili, G., and Maison, D., "A remark on the instability of the Bartnik-McKinnon solutions", Phys. Lett. B, 343, 214-217, (1995).

[137] Lechner, C., Staticity, self-similarity and critical phenomena in a self-gravitating nonlinear sigma model, (University of Vienna, Vienna, 2001). Related online version (cited on 15 June 2007):

http://arXiv.org/abs/gr-qc/0507009

[138] Lechner, C., Thornburg, J., Husa, S., and Aichelburg, P.C., "A new transition between discrete and continuous self-similarity in critical gravitational collapse", Phys. Rev. D, 65, 081501, (2002). Related online version (cited on 1 February 2003): http://arXiv.org/abs/gr-qc/0112008.

[139] Levin, J., "Gravity waves, chaos, and spinning compact binaries", Phys. Rev. Lett., 84, 3515, (2000). Related online version (cited on 15 June 2007): http://arXiv.org/abs/gr-qc/9910040.

[140] Liebling, S.L., "Multiply unstable black hole critical solutions", Phys. Rev. D, 58, 0840151-8, (1998). Related online version (cited on 10 December 1999): http://arXiv.org/abs/gr-qc/9805043.

[141] Liebling, S.L., "Critical phenomena inside global monopoles", Phys. Rev. D, 60, 061502, (1999). Related online version (cited on 10 December 1999): http://arXiv.org/abs/gr-qc/9904077.

[142] Liebling, S.L., "The singularity threshold of the nonlinear sigma model using 3D adaptive mesh refinement", Phys. Rev. D, 66, 041703, (2002). Related online version (cited on 1 February 2003): http://arXiv.org/abs/gr-qc/0202093 
[143] Liebling, S.L., and Choptuik, M.W., "Black hole criticality in the Brans-Dicke model", Phys. Rev. Lett., 77, 1424-1427, (1996). Related online version (cited on 10 December 1999): http://arXiv.org/abs/gr-qc/9606057.

[144] Liebling, S.L., Hirschmann, E.W., and Isenberg, J., "Critical phenomena in nonlinear sigma models", J. Math. Phys., 41, 5691, (2000). Related online version (cited on 1 February 2003): http://arXiv.org/abs/math-ph/9911020.

[145] Maison, D., "Non-universality of critical behaviour in spherically symmetric gravitational collapse", Phys. Lett. B, 366, 82-84, (1996). Related online version (cited on 10 December 1999): http://arXiv.org/abs/gr-qc/9504008

[146] Martín-García, J.M., and Gundlach, C., "All nonspherical perturbations of the Choptuik spacetime decay", Phys. Rev. D, 59, 064031, (1999). Related online version (cited on 10 December 1999): http://arXiv.org/abs/gr-qc/9809059

[147] Martín-García, J.M., and Gundlach, C., "Static and self-similar spherically symmetric solutions of the Einstein-Vlasov system", Phys. Rev. D, 65, 084026, (2002). Related online version (cited on 1 February 2003): http://arXiv.org/abs/gr-qc/0112009

[148] Martín-García, J.M., and Gundlach, C., "Critical Phenomena in Gravitational Collapse: The Role of Angular Momentum", in Fernández-Jambrina, L., and González-Romero, L.M., eds., Current Trends in Relativistic Astrophysics, Spanish Relativity Meeting, held in September 2001, in Madrid, Spain, 68-86, (Lecture Notes in Physics 617, Springer-Verlag, Berlin, 2003).

[149] Martín-García, J.M., and Gundlach, C., "Global structure of Choptuik's critical solution in scalar field collapse", Phys. Rev. D, 68, 024011, (2003). Related online version (cited on 1 February 2003): http://arXiv.org/abs/gr-qc/0304070

[150] Millward, R.S., and Hirschmann, E.W., "Critical behavior of gravitating sphalerons", Phys. Rev. D, 68, 024017, (2003). Related online version (cited on 15 June 2007): http://arXiv.org/abs/gr-qc/0212015.

[151] Musco, I., Miller, J.C., and Rezzolla, L., "Computations of primordial black hole formation", Class. Quant. Grav, 22, 1405-1424, (2005). Related online version (cited on 15 June 2007): http://arXiv.org/abs/gr-qc/0412063

[152] Neilsen, D.W., and Choptuik, M.W., "Critical phenomena in perfect fluids", Class. Quant. Grav., 17, 761-782, (2000). Related online version (cited on 10 December 1999): http://arXiv.org/abs/gr-qc/9812053

[153] Neilsen, D.W., and Choptuik, M.W., "Ultrarelativistic fluid dynamics", Class. Quant. Grav., 17, 733-759, (2000). Related online version (cited on 10 December 1999): http://arXiv.org/abs/gr-qc/9904052

[154] Niemeyer, J.C., and Jedamzik, K., "Near-Critical Gravitational Collapse and the Initial Mass Function of Primordial Black Holes", Phys. Rev. Lett., 80, 5481-5484, (1998). Related online version (cited on 10 December 1999): http://arXiv.org/abs/gr-qc/9709072 
[155] Niemeyer, J.C., and Jedamzik, K., "Dynamics of primordial black hole formation", Phys. Rev. D, 59, 124013, (1999). Related online version (cited on 10 December 1999): http://arXiv.org/abs/astro-ph/9901292.

[156] Noble, S.C., A numerical study of relativistic fluid collapse, (University of Texas at Austin, Austin, 2003). Related online version (cited on 15 June 2007): http://arXiv.org/abs/gr-qc/0310116.

[157] Noble, S.C., and Choptuik, M.W., "Type II critical phenomena of neutron star collapse", (2007). Related online version (cited on 27 November 2007): http://arXiv.org/abs/0709.3527.

[158] Novak, J., "Velocity-induced collapses of stable neutron stars", Astron. Astrophys., 376, 606, (2001). Related online version (cited on 1 February 2003): http://arXiv.org/abs/gr-qc/0107045.

[159] Olabarrieta, I., and Choptuik, M. W., "Critical phenomena at the threshold of black hole formation for collisionless matter in spherical symmetry", Phys. Rev. D, 65, 024007, (2002). Related online version (cited on 1 February 2003): http://arXiv.org/abs/gr-qc/0107076.

[160] Oliveira-Neto, G., and Takakura, F.I., "Wyman's solution, self-similarity and critical behaviour", J. Math. Phys., 46, 062503, (2005). Related online version (cited on 15 June 2007): http://arXiv.org/abs/gr-qc/0309099

[161] Ori, A., and Piran, T., "Naked singularities in self-similar spherical gravitational collapse", Phys. Rev. Lett., 59, 2137, (1987).

[162] Ori, A., and Piran, T., "Naked singularities and other features of self-similar generalrelativistic gravitational collapse", Phys. Rev. D, 42, 1068-1090, (1990).

[163] Oshiro, Y., Nakamura, K., and Tomimatsu, A., "Critical behavior of black hole formation in a scalar wave Collapse", Prog. Theor. Phys., 91, 1265-1270, (1994). Related online version (cited on 10 December 1999): http://arXiv.org/abs/gr-qc/9402017.

[164] Peleg, Y., Bose, S., and Parker, L., "Choptuik scaling and quantum effects in 2D dilaton gravity", Phys. Rev. D, 55, 4525-4528, (1997). Related online version (cited on 10 December 1999): http://arXiv.org/abs/gr-qc/9608040.

[165] Peleg, Y., and Steif, A., "Phase transition for gravitationally collapsing dust shells in 2+1 dimensions", Phys. Rev. D, 51, 3992-3996, (1995). Related online version (cited on 1 February 2003): http://arXiv.org/abs/gr-qc/9412023.

[166] Petryk, R., Maxwell-Klein-Gordon fields in black hole spacetimes, (University of British Columbia, Vancouver, 2006). Available from http://laplace.physics.ubc.ca/People/matt/Doc/Theses/.

[167] Pretorius, F., "Black hole mergers and unstable circular orbits", Class. Quant. Grav., 24, S83-S108, (2007). Related online version (cited on 1 February 2003): http://arXiv.org/abs/gr-qc/0702084. 
[168] Pretorius, F., and Choptuik, M.W., "Gravitational collapse in 2+1 dimensional AdS spacetime", Phys. Rev. D, 62, 124012, (2000). Related online version (cited on 1 February 2003): http://arXiv.org/abs/gr-qc/0007008.

[169] Price, R.H., and Pullin, J., "Analytic approximations to the spacetime of a critical gravitational collapse", Phys. Rev. D, 54, 3792-3799, (1996). Related online version (cited on 10 December 1999): http://arXiv.org/abs/gr-qc/9601009

[170] Pullin, J., "Is there a connection between no-hair behavior and universality in gravitational collapse?", Phys. Lett. A, 204, 7, (1995). Related online version (cited on 10 December 1999): http://arXiv.org/abs/gr-qc/9409044.

[171] Pürrer, M., Husa, S., and Aichelburg, P.C., "News from Critical Collapse: Bondi Mass, Tails and Quasinormal Modes", Phys. Rev. D, 71, 104005, (2005). Related online version (cited on 15 June 2007): http://arXiv.org/abs/gr-qc/0411078.

[172] Rein, G., Rendall, A.D., and Schaeffer, J., "Critical collapse of collisionless matter: A numerical investigation", Phys. Rev. D, 58, 044007-1-8, (1998). Related online version (cited on 10 December 1999): http://arXiv.org/abs/gr-qc/9804040

[173] Roberts, M.D., "Scalar field counterexamples to the cosmic censorship hypothesis", Gen. Relativ. Gravit., 21, 907-939, (1989).

[174] Sarbach, O., and Lehner, L., "Critical bubbles and implications for critical black strings", Phys. Rev. D, 71, 026002, (2005). Related online version (cited on 15 June 2007): http://lanl.arxiv.org/abs/hep-th/0407265

[175] Seidel, E., and Suen, W.-M., "Oscillating soliton stars", Phys. Rev. Lett., 66, 1659, (1991).

[176] Smarr, L., and York Jr., J.W., "Kinematical conditions in the construction of spacetime", Phys. Rev. D, 17, 2529, (1978).

[177] Snajdr, M., "Critical collapse of an ultrarelativistic fluid in the $\Gamma \rightarrow 1$ limit", Class. Quant. Grav., 23, 6857-6872, (2006). Related online version (cited on 15 June 2007): http://arXiv.org/abs/gr-qc/0508062.

[178] Sorkin, E., and Oren, Y., "On Choptuik's scaling in higher dimensions", Phys. Rev. D, 71, 124005, (2005). Related online version (cited on 15 June 2007): http://arXiv.org/abs/gr-qc/

[179] Stevenson, R., The spherically symmetric collapse of collisionless matter. Exploring critical phenomena through finite volume methods., (University of British Columbia, Vancouver, 2005). Related online version (cited on 15 June 2007): http://laplace.physics.ubc.ca/Theses/Msc/stevenson.ps.

[180] Straumann, N., and Zhou, Z.H., "Instability of a colored black hole solution", Phys. Lett. B, 243, 33-35, (1990).

[181] Strominger, A., and Thorlacius, L., "Universality and scaling at the onset of quantum black hole formation", Phys. Rev. Lett., 72, 1584-1587, (1994). Related online version (cited on 10 December 1999): http://arXiv.org/abs/hep-th/9312017. 
[182] Szybka, S.J., "Chaotic self-similar wave maps coupled to gravity", Phys. Rev. D, 69, 084014, (2004). Related online version (cited on 15 June 2007):

http://arXiv.org/abs/gr-qc/0310050.

[183] Thornburg, J.and Lechner, C., Pürrer, M., Aichelburg, P.C., and Husa, S., "Episodic selfsimilarity in critical gravitational collapse", in Proceedings of the 9th Marcel Grossman Meeting, Marcel Grossman Meeting. World Scientific, (2001). Related online version (cited on 1 February 2003):

http://arXiv.org/abs/gr-qc/0012043.

[184] Tod, P., personal communication.

[185] van Putten, M.H.P.M., "Approximate black holes for numerical relativity", Phys. Rev. D, 54, R5931, (1996). Related online version (cited on 15 June 2007): http://arXiv.org/abs/gr-qc/9607074.

[186] Ventrella, J.F., and Choptuik, M.W., "Critical Phenomena in the Einstein-Massless-Dirac System", Phys. Rev. D, 68, 044020, (2003). Related online version (cited on 15 June 2007): http://arXiv.org/abs/gr-qc/0304007.

[187] Volkov, M.S., Brodbeck, O., Lavrelashvili, G., and Straumann, N., "The number of sphaleron instabilities of the Bartnik-McKinnon solitons and", Phys. Lett. B, 349, 438-442, (1995). Related online version (cited on 10 December 1999): http://arXiv.org/abs/hep-th/9502045.

[188] Volkov, M.S., and Gal'tsov, D.V., "Non-abelian Einstein-Yang-Mills black holes", J. Exp. Theor. Phys. Lett., 50, 346-350, (1990).

[189] Wald, R.M., "Gravitational Collapse and Cosmic Censorship", (October, 1997). Related online version (cited on 10 December 1999):

http://arXiv.org/abs/gr-qc/9710068

[190] Wang, A., "Critical collapse of cylindrically symmetric scalar field in four-dimensional Einstein's theory of gravity", Phys. Rev. D, 68, 064006, (2003). Related online version (cited on 15 June 2007): http://arXiv.org/abs/gr-qc/0307071.

[191] Wang, A., and de Oliveira, H.P., "Critical phenomena of collapsing massless scalar wave packets", Phys. Rev. D, 56, 753-761, (1997). Related online version (cited on 10 December 1999): http://arXiv.org/abs/gr-qc/9608063

[192] Wyman, M., "Static spherically symmetric scalar fields in general relativity", Phys. Rev. D, 19, 839, (2002). Related online version (cited on 1 February 2003): http://arXiv.org/abs/gr-qc/0111039.

[193] Yeomans, J.M., Statistical Mechanics of Phase Transitions, Oxford Science Publications, (Clarendon Press; Oxford University Press, Oxford, U.K.; New York, U.S.A., 1992).

[194] Yokoyama, J., "Cosmological constraints on primordial black holes produced in the nearcritical gravitational collapse", Phys. Rev. D, 58, 107502, (1998). Related online version (cited on 10 December 1999): http://arXiv.org/abs/gr-qc/9804041. 
[195] Zhou, J.-G., Müller-Kirsten, H.J.W., and Yang, M.-Z., "New look at the critical behaviour near the threshold of black hole formation in the Russo-Susskind-Thorlacius model", Phys. Rev. D, 51, R314, (1995). 